This item was submitted to Loughborough's Research Repository by the author.

Items in Figshare are protected by copyright, with all rights reserved, unless otherwise indicated.

\title{
Finite-barrier corrections for multidimensional barriers in colored noise
}

PLEASE CITE THE PUBLISHED VERSION

https://doi.org/10.1103/physreve.99.052211

\section{PUBLISHER}

(C) American Physical Society (APS)

\section{VERSION}

VoR (Version of Record)

\section{PUBLISHER STATEMENT}

This work is made available according to the conditions of the Creative Commons Attribution-NonCommercialNoDerivatives 4.0 International (CC BY-NC-ND 4.0) licence. Full details of this licence are available at: https://creativecommons.org/licenses/by-nc-nd/4.0/

\section{LICENCE}

CC BY-NC-ND 4.0

\section{REPOSITORY RECORD}

Bartsch, Thomas, F. Revuelta, R.M. Benito, and F. Borondo. 2019. "Finite-barrier Corrections for Multidimensional Barriers in Colored Noise". figshare. https://hdl.handle.net/2134/37807. 


\title{
Finite-barrier corrections for multidimensional barriers in colored noise
}

\author{
Thomas Bartsch, ${ }^{1, *}$ F. Revuelta, ${ }^{2,3, \dagger}$ R. M. Benito, ${ }^{2, \ddagger}$ and F. Borondo ${ }^{3,4,8}$ \\ ${ }^{1}$ Centre for Nonlinear Mathematics and Applications, Department of Mathematical Sciences, Loughborough University, \\ Loughborough LE11 3TU, England, United Kingdom \\ ${ }^{2}$ Grupo de Sistemas Complejos, E. T. S. Ingeniería Agronómica, Alimentaria y de Biosistemas, Universidad Politécnica de Madrid, \\ Avenida Puerta de Hierro 2-4, 28040 Madrid, Spain \\ ${ }^{3}$ Instituto de Ciencias Matemáticas, Cantoblanco, 28049 Madrid, Spain \\ ${ }^{4}$ Departamento de Química, Universidad Autónoma de Madrid, Cantoblanco, 28049 Madrid, Spain
}

(Received 4 August 2018; published 15 May 2019)

\begin{abstract}
The usual identification of reactive trajectories for the calculation of reaction rates requires very timeconsuming simulations, particularly if the environment presents memory effects. In this paper, we develop a method that permits the identification of reactive trajectories in a system under the action of a stochastic colored driving. This method is based on the perturbative computation of the invariant structures that act as separatrices for reactivity. Furthermore, using this perturbative scheme, we have obtained a formally exact expression for the reaction rate in multidimensional systems coupled to colored noisy environments.
\end{abstract}

DOI: 10.1103/PhysRevE.99.052211

\section{INTRODUCTION}

Transition state theory (TST) is one of the most successful and widespread theories of chemistry. Ever since its inception [1-4] TST has provided a powerful conceptual framework for reaction rate theory. It has been applied to a wide variety of activated processes, that proceed from suitably defined "reactant" to "product" states [5-14] in which the rate limiting step is the crossing of an energetic barrier.

In this situation the vast majority of reactive trajectories will pass very close to the top of the barrier. If a dividing surface (DS) between reactant and product regions of phase space is chosen close to the barrier top, the reaction rate can be computed from the steady-state flux of trajectories through this surface. To this end, TST makes two simplifying assumptions: first, that every trajectory that crosses the DS from the reactant to the product side is reactive or, equivalently, that no trajectory can cross the DS more than once, and, second, that in the steady state the reactants follow a thermal equilibrium distribution near the barrier.

If either of these assumptions is violated, suitable corrections need to be introduced into TST. For a reactive system coupled to a thermal bath, the no-recrossing assumption poses particular challenges because, unless a DS is sought in an infinite-dimensional extended phase space [15], the randomly fluctuating force exerted by the bath will cause the system to cross any chosen DS potentially many times, in particular in strongly damped systems. Consequently, a recrossing-free DS cannot be found in thermal systems with anharmonic barriers [16] (or even for gas phase reactions at high energies [17-19]). It is this challenge that we propose to address in this paper, in

\footnotetext{
*T.Bartsch@lboro.ac.uk

†fabio.revuelta@upm.es

†rosamaria.benito@upm.es

§f.borondo@uam.es
}

a manner that is appropriate for systems with many degrees of freedom (DOF's) and for heat baths that are modeled by white (memoryless) as well as colored (correlated) noise, i.e., in the presence of Markovian and non-Markovian friction. An application to the realistic isomerizing reaction $\mathrm{LiNC} \rightleftharpoons \mathrm{LiCN}$ in the presence of an argon bath using a one-dimensional approach can be found in Ref. [20].

A widely used model for the interaction of a reactive system with a surrounding heat bath is the Langevin equation (LE) [21-23]. It neglects quantum effects such as barrier tunneling, which can be important in the case of light particles [24], and the interaction with electronic excited states through conical intersections [25]. More importantly for our purposes, it assumes that the random force exerted by the bath is not correlated with itself or with the reactive system, and that the collisions with the bath are instantaneous. A more realistic model of a heat bath will take into account that the stochastic forces that the bath exerts on the reactive system at different times must be correlated, and this correlation will decay on a time scale that is given by the dynamics of the bath. This effect can be described by a generalized Langevin equation (GLE) (see Refs. [21-23,26] and Sec. III below).

The phase space of the GLE has infinite dimension. However, if the friction kernel satisfies a linear differential equation with constant coefficients, the GLE can be rephrased as a stochastic differential equation on a finite-dimensional phase space [27]. The methods proposed in this paper apply to kernels of this type, though for simplicity only an exponential friction kernel will be discussed directly [see Eq. (12) below]. The permissible class of friction kernels encompasses a wide range of friction kernels of practical importance. Furthermore, any friction kernel that tends to zero for large times can be approximated by such kernels to arbitrary accuracy according to the Stone-Weierstrass theorem [28].

Rate theories are usually derived under the assumption that the height of the barrier is much greater than the thermal 
energy $k_{\mathrm{B}} T$. At higher temperatures deviations from the highbarrier limit can be noticeable. Finite-barrier corrections to the rates were obtained by Drozdov and Talkner in Ref. [29] for systems with white noise, and corrections to the transmission factor were obtained by Pollak and Talkner in Refs. [30-32] for systems with either white or correlated noise. Corrections to the depopulation factor are available in recent extensions to turnover theory [33-36]. Different methods have been developed to account for the previous effects. For example, some theories were based on the Rayleigh method [29], while others [30-32] were based on the equivalence between the original LE as well as its generalization for correlated noise to a Hamiltonian model in which the reactive system is coupled to an infinite bath of harmonic oscillators [37]. Additionally, these results are mostly restricted to one-dimensional systems. For example, Ref. [38] computes the reaction rates of a onedimensional potential with a quartic anharmonicity driven by colored noise by choosing a planar dividing surface that minimizes the reactive flux, as usual within the variational TST.

It is the purpose of the present paper to present a general method by which rate corrections in multidimensional systems can be derived. We will focus on corrections of the transmission factor. This is, in practice, not a severe restriction since most reactions occur in the regime of moderate to high friction. In fact, to the best of our knowledge a reaction that shows the Kramers turnover in a realistic setting was first demonstrated in Ref. [39].

Moreover, we avoid using an explicit model of the heat bath that introduces an infinite-dimensional phase space. Instead, we work directly in the phase space of the GLE, which is finite dimensional for the friction kernels we consider. This choice is convenient from both computational and conceptual points of view, since it allows one to visualize the relevant phase space structures more easily. The central ingredient of this paper is a detailed description of the geometrical structures that determine reactivity in the phase space of the multidimensional GLE. This paper is based on a recent series of papers [20,40-46] that develop such structures and their use in rate theory in the spatial diffusion regime. The previous papers were mostly restricted to the LE with white noise. They show the existence of a particular trajectory that remains in the vicinity of the barrier top for all times, without ever descending into either well. It is called the transition state trajectory and depends on the realization of the noise. For the case of a harmonic barrier, the GLE becomes noiseless if the dynamics is studied in a time-dependent coordinate system with the TS trajectory as the origin. It is then easy to identify hypersurfaces that separate reactive from nonreactive trajectories. The most important of these is the stable manifold of the TS trajectory. It contains all trajectories that asymptotically approach the TS trajectory for long times and it separates trajectories that descend into the product well in the distant future from those that descend into the reactant well. A knowledge of the stable manifold therefore allows one to distinguish reactive from nonreactive trajectories without any further computation. It solves the diagnostic problem that is fundamental to rate theory.

The stable manifold will persist if the barrier is not harmonic. In Refs. [45,46] we demonstrated how it can be computed by perturbation theory in the presence of white noise. We will demonstrate here in detail that the stable manifold also exists in a reactive system described by a GLE with Gaussian correlated noise, and we will use it to derive anharmonic barrier corrections to the reaction rate for such systems, even when they have many DOF's. We will obtain leading order rate corrections for a generic one-dimensional barrier and a potential $[42,43,45,46]$ with two DOF's that already present all the complexity of systems of higher dimensionality.

The outline of the paper is as follows. In Sec. II we introduce the fundamentals of the rate theory that are necessary for our purposes. Section III presents the GLE and its phase space coordinates. The geometrical structures that characterize the phase space and are central for our paper are described in Sec. IV. Section V is devoted to the calculation of a critical velocity that allows a unique identification of reactive trajectories. In Sec. VI we explain how this critical velocity can be used for the calculation of the transmission factor. Section VII presents and discusses the results of our paper. Finally, we summarize in Sec. VIII the conclusions of our paper.

\section{THE FUNDAMENTAL RATE FORMULA}

In this section, we summarize the fundamentals of reaction rate theory that will be used in the rest of the paper. For a more detailed discussion, see, for example, Refs. [21-23].

Let us consider a system with $d$ DOF's described by the $d$-dimensional coordinate vector $\boldsymbol{x}$ the components of which will be denoted by $x_{n}$, with $n=0,1, \ldots, d-1$, where $n=0$ denotes the reactive mode and $n=1, \ldots, d-1$ correspond to the bath modes. If a DS is placed at a certain value $x_{0}=x_{0}^{\mathrm{DS}}$, and the reactant and product regions are defined by $x_{0}<x_{0}^{\mathrm{DS}}$ and $x_{0}>x_{0}^{\mathrm{DS}}$, respectively, the reaction rate is given by the flux-over-population expression

$$
k=\frac{J}{N},
$$

where $N$ is the average population of the reactant region and $J$ is the reactive flux out of it. The DS $x_{0}=x_{0}^{\mathrm{DS}}$ can be parametrized by $2 d-1$ phase space coordinates: the velocity $v_{0}$ perpendicular to the surface and the coordinates $\boldsymbol{x}_{\perp}=$ $\left(x_{1}, x_{2}, \ldots, x_{d-1}\right)$, and velocities $v_{\perp}=\left(v_{1}, v_{2}, \ldots, v_{d-1}\right)$ in the transverse directions. The reactive flux then equals

$$
J=\left\langle v_{0} \chi_{\alpha}\left(v_{0}, \boldsymbol{x}_{\perp}, v_{\perp}\right)\right\rangle_{\alpha, \mathrm{IC}}
$$

where the average extends over all realizations $\alpha$ of the noise and over a stationary-state ensemble of initial conditions (IC's) on the DS. [In the definition of Eq. (2) of the reactive flux we have omitted irrelevant constant factors that will cancel in the transmission factor of Eq. (5).] The characteristic function $\chi_{\alpha}$ takes the value 1 if the trajectory given by the IC $\left(x_{0}^{\mathrm{DS}}, v_{0}, \boldsymbol{x}_{\perp}, v_{\perp}\right.$ ) is reactive if driven by the noise sequence $\alpha$ and zero otherwise. It ensures that a trajectory is only included in the reactive flux if it actually leads to a reaction, i.e., if it descends from the barrier into the product region and thermalizes there. We will propose below a simple explicit expression for $\chi_{\alpha}$ that incorporates all the potentially intricate dynamics of the system. 
Standard TST sidesteps the dynamical problem by assuming that the DS is recrossing free and that a trajectory that crosses from reactant to product is therefore reactive. In other words, TST assumes the characteristic function $\chi^{\mathrm{TST}}\left(v_{0}\right)=$ $\Theta\left(v_{0}\right)$, the Heaviside step function. The corresponding flux

$$
J^{\mathrm{TST}}=\left\langle v_{0} \chi^{\mathrm{TST}}\left(v_{0}\right)\right\rangle_{v_{0}}
$$

leads to an approximation to the reaction rate,

$$
k^{\mathrm{TST}}=\frac{J^{\mathrm{TST}}}{N},
$$

that always overestimates the true rate. The extend to which a given system violates the no-recrossing assumption is measured by the transmission factor

$$
\kappa=\frac{k}{k^{\mathrm{TST}}}<1 .
$$

Unless the friction caused by the heat bath is very weak, the stationary-state distribution of IC's in the barrier is given by a Boltzmann equilibrium distribution. This assumption will always be made in the rate calculations presented here, though the dynamical theory at the heart of this paper does not require it because the exact expression for the characteristic function applies to equilibrium as well as nonequilibrium systems. The average over IC's is then performed over an ensemble with probability density

$$
p\left(x_{0}, v_{0}, \boldsymbol{x}_{\perp}, v_{\perp}\right)=\delta\left(x_{0}-x_{0}^{\mathrm{DS}}\right) \exp \left(-\frac{m v_{0}^{2}}{2 k_{\mathrm{B}} T}\right) p_{\perp},
$$

where $m$ is the particle mass and $p_{\perp}=p_{\perp}\left(\boldsymbol{x}_{\perp}, v_{\perp}\right)$ is a Boltzmann distribution

$$
p_{\perp}\left(\boldsymbol{x}_{\perp}, v_{\perp}\right)=\frac{1}{Z} \exp \left(-\frac{m v_{\perp}^{2} / 2+U\left(x_{0}^{\mathrm{DS}}, \boldsymbol{x}_{\perp}\right)}{k_{\mathrm{B}} T}\right)
$$

for the transverse coordinates and velocities, $U\left(x_{0}^{\mathrm{DS}}, \boldsymbol{x}_{\perp}\right)$ being the potential of mean force and $Z$ being the partition function that ensures $\int d \boldsymbol{x}_{\perp} d v_{\perp} p_{\perp}\left(\boldsymbol{q}_{\perp}, v_{\perp}\right)=1$. Under this assumption, the TST flux given by Eq. (3) can be evaluated analytically to give

$$
J^{\mathrm{TST}}=\sqrt{\frac{k_{\mathrm{B}} T}{2 \pi m}} .
$$

\section{THE GENERALIZED LANGEVIN EQUATION}

The dynamics of a $d$ DOF system coupled to an external heat bath that has memory effects [38] can be accurately described by the GLE

$$
m \ddot{\boldsymbol{x}}=-\nabla_{\boldsymbol{x}} U(\boldsymbol{x})-m \int_{-\infty}^{t} \boldsymbol{\Gamma}(t-s) \dot{\boldsymbol{x}}(s) d s+m \boldsymbol{R}_{\alpha}(t),
$$

where $\boldsymbol{\Gamma}(t)$ is the friction kernel $d \times d$ matrix and $\boldsymbol{R}_{\alpha}(t)$ is the fluctuating Gaussian noise force exerted by the heat bath. The correlation function of the components of $\boldsymbol{R}_{\alpha}(t)$ is given by the fluctuation-dissipation theorem

$$
\left\langle R_{\alpha, n}(0) R_{\alpha, n^{\prime}}(t)\right\rangle_{\alpha}=\frac{k_{\mathrm{B}} T \boldsymbol{\Gamma}(t)}{m} \delta_{n n^{\prime}},
$$

where $n, n^{\prime}=0,1, \ldots, d-1$, and $\langle\ldots\rangle_{\alpha}$ denotes an average over the different realizations $\alpha$ of the noise.
With no loss of generality, we will assume that the top of the energetic barrier that separates reactants and products is located at $\boldsymbol{x}=\mathbf{0}$. Recall that it is at this point where a bottleneck for reactivity is formed, and in the regime of moderate to strong friction the shape of the potential in this region determines the rate. Specifically, the relevant neighborhood of the saddle point, which in our approach is represented by the area explored by the TS trajectory, has an extension of order $\sqrt{k_{\mathrm{B}} T}$, as will be seen in Sec. VII. Thus, if the potential is expanded as a Taylor series around the saddle point, each order can be regarded as being smaller than the preceding order by a factor $\sqrt{k_{\mathrm{B}} T}$. We will below introduce a formal expansion parameter $\varepsilon$ to reflect this ordering. This is merely a book-keeping device; the final rate formulas will depend on the coefficients of the Taylor expansion.

A fundamental assumption of rate theory is that the thermal energy $k_{\mathrm{B}} T$ must be small compared to the barrier height $V^{\ddagger}$, measured from the bottom of the reactant well to the barrier top. A rate correction can sometimes be written as an expansion in the small parameter $k_{\mathrm{B}} T / V^{\ddagger}$, as was done, for example, in Refs. [29-31]. However, the barrier height is a nonlocal feature of the potential, and in a complicated potential the various expansion coefficients of the potential cannot all be expressed by a single small parameter. In the regime of moderate to strong friction the reaction rate is determined by spatial diffusion in the neighborhood of the barrier, and the Taylor coefficients directly characterize this neighborhood. We therefore choose to express the rate corrections in terms of the expansion coefficients, even where it would be possible to rewrite the result in terms of the barrier height.

In the expansion of the potential the term of order zero, i.e., the value $U(\mathbf{0})$, can be set to zero without loss of generality. The first order terms vanish because the expansion is around a saddle point. We choose a coordinate system in which the harmonic part of the potential is diagonal. The mean force can then be split into harmonic and anharmonic contributions as

$$
-\frac{\partial U\left(x_{n}\right)}{\partial x_{n}}=\sigma_{n} m \omega_{n}^{2} x_{n}+m f_{n}(\boldsymbol{x}),
$$

where $\sigma_{n}=+1$ for $n=0$ and $\sigma_{n}=-1$ for $1 \leqslant n \leqslant d-1$ mark the difference between the reactive mode and the bath modes, and $f_{n}(\boldsymbol{x})$ denotes the anharmonic terms. Notice that Eq. (11) is valid for all potential energy surfaces with a barrier top that has a saddle $\times$ center $\times$ center $\times \ldots \times$ center structure.

\section{A. The extended phase space}

In this paper, we assume an isotropic exponential friction kernel

$$
\boldsymbol{\Gamma}(t)=\gamma(t) \mathbf{I}, \quad \text { with } \gamma(t)=\frac{\gamma_{0}}{\tau} e^{-t / \tau},
$$

a characteristic correlation time $\tau$, and a damping strength $\gamma_{0}$. This kernel couples all DOF's to the heat bath with equal strength. It accurately describes the behavior of many realistic chemical reactions [39]. In this case, as for a variety of other friction kernels, the GLE given by Eq. (9), which is a complicated integrodifferential equation, can be replaced by a system of differential equations on a finite-dimensional extended phase space $[27,38,47-49]$ with the auxiliary 
coordinates

$$
\zeta_{n}=-\int_{-\infty}^{t} \gamma(t-s) \dot{x}_{n}(s) d s
$$

On the extended phase space, the GLE with exponential friction can be represented by the system of differential equations

$$
\begin{aligned}
& \dot{x}_{n}=v_{n}, \\
& \dot{v}_{n}=-\frac{1}{m} \frac{\partial U(\boldsymbol{x})}{\partial x_{n}}+\zeta_{n}, \\
& \dot{\zeta}_{n}=-\frac{\gamma_{0}}{\tau} v_{n}-\frac{1}{\tau} \zeta_{n}+\xi_{\alpha, n}(t),
\end{aligned}
$$

now with a white noise source vector $\boldsymbol{\xi}_{\alpha}$ acting on all DOF's and satisfying the fluctuation-dissipation theorem

$$
\left\langle\xi_{\alpha, n}(t) \xi_{\alpha, n^{\prime}}(s)\right\rangle_{\alpha}=\frac{2 k_{\mathrm{B}} T \gamma_{0}}{m \tau^{2}} \delta_{n n^{\prime}} \delta(t-s) .
$$

In the definition of the auxiliary coordinate given by Eq. (13), the choice of $-\infty$ as the lower limit of integration represents the assumption that the system was prepared in the infinite past. This assumption is essential to guarantee that the phase space is indeed the $3 d$-dimensional space with coordinates $\boldsymbol{x}, \boldsymbol{v}$, and $\boldsymbol{\zeta}$, rather than a submanifold thereof [50]. In thermal equilibrium, the auxiliary coordinates follow a Gaussian distribution with zero mean correlation $\left\langle\zeta_{n} \zeta_{n^{\prime}}\right\rangle=$ $\frac{k_{\mathrm{B}} T \gamma_{0}}{m \tau} \delta_{n, n^{\prime}}$. It is not correlated with either position or velocity [49]. Accordingly, in the rate calculation, the average over IC's in Eq. (2) must be supplemented by an average over the distribution of the auxiliary coordinates.

\section{B. Dynamics near a harmonic barrier}

In the harmonic approximation, i.e., $f_{n}(\boldsymbol{x})=0$, and temporarily neglecting the noise, the equations of motion (EoM) (14) can be rewritten as

$$
\dot{\boldsymbol{u}}_{n}=\mathbf{M}_{n} \boldsymbol{u}_{n}
$$

with

$$
\mathbf{M}_{n}=\left(\begin{array}{ccc}
0 & 1 & 0 \\
\sigma_{n} \omega_{n}^{2} & 0 & 1 \\
0 & -\frac{\gamma_{0}}{\tau} & -\frac{1}{\tau}
\end{array}\right)
$$

and

$$
\boldsymbol{u}_{n}=\left(\begin{array}{c}
x_{n} \\
v_{n} \\
\zeta_{n}
\end{array}\right)
$$

The eigenvalues $\lambda_{n, 0}, \lambda_{n, 1}$, and $\lambda_{n, 2}$ of the matrix $\mathbf{M}_{\mathbf{n}}$, obtained as the zeros of the characteristic polynomial

$$
P_{n}(\lambda)=-\lambda^{3}-\frac{1}{\tau} \lambda^{2}+\left[\sigma_{n} \omega_{n}^{2}-\frac{\gamma_{0}}{\tau}\right] \lambda+\sigma_{n} \frac{\omega_{n}^{2}}{\tau},
$$

are, in general, different. The corresponding eigenvectors are

$$
\tilde{\boldsymbol{u}}_{n, i}=\left(\begin{array}{c}
1 \\
\lambda_{n, i} \\
\lambda_{n, i}^{2}+\sigma_{n} \omega_{n}^{2}
\end{array}\right)
$$

Algebraic expressions for the eigenvalues could in principle be given, but they are unwieldy. More useful are the Vieta relations obeyed by the eigenvalues

$$
\begin{aligned}
\lambda_{n, 0}+\lambda_{n, 1}+\lambda_{n, 2} & =-\frac{1}{\tau}, \\
\lambda_{n, 0} \lambda_{n, 1}+\lambda_{n, 0} \lambda_{n, 2}+\lambda_{n, 1} \lambda_{n, 2} & =\frac{\gamma_{0}}{\tau}-\sigma_{n} \omega_{n}^{2}, \\
\lambda_{n, 0} \lambda_{n, 1} \lambda_{n, 2} & =\sigma_{n} \frac{\omega_{n}^{2}}{\tau} .
\end{aligned}
$$

As $P_{0}(0)=\omega_{0}^{2} / \tau>0$ and $P_{0}\left(\omega_{0}\right)=-\gamma_{0} \omega_{0} / \tau<0$, at least one of the eigenvalues, say $\lambda_{0,0}$, must be real and lie between zero and $\omega_{0}$. This eigenvalue describes an unstable direction in phase space. The remaining eigenvalues have all real negative parts, and, as a consequence, they correspond to stable directions in the extended phase space. At least one in every DOF, say $\lambda_{n, 0}$, is always real and lies between $-1 / \tau$ and zero, as $P_{n}(0)=-\omega_{n}^{2} / \tau<0$ and $P_{n}(-1 / \tau)=\gamma_{0} / \tau^{2}>0$ for $n \geqslant 1$. Finally, the remaining eigenvalues, $\lambda_{n, 1}$ and $\lambda_{n, 2}$, must either be both real and negative or form a complex conjugate pair with real negative parts since, according to the Vieta relations in Eq. (21), $\lambda_{n, 1}+\lambda_{n, 2}=-1 / \tau-\lambda_{n, 0}<0$ and $\lambda_{n, 1} \lambda_{n, 2}=\sigma_{n} \omega_{n}^{2} /\left(\tau \lambda_{n, 0}\right)>0$. In either case, a trajectory will approach the origin in these directions as $t \rightarrow \infty$, either in an oscillatory manner (if $\lambda_{n, 1}$ and $\lambda_{n, 2}$ are complex) or monotonically (otherwise). The boundary between these different types of behavior in parameter space is given by the condition $\lambda_{n, 1}=\lambda_{n, 2}$. As a consequence, the matrix $\mathbf{M}_{\mathbf{n}}$ is not diagonalizable; it is easy to check that Eq. (20) is the only solution of the eigenvector equation $\mathbf{M}_{\mathbf{n}} \tilde{\boldsymbol{u}}_{\boldsymbol{n}, i}=\lambda_{n, i} \tilde{\boldsymbol{u}}_{\boldsymbol{n}, \boldsymbol{i}}$ for any given eigenvalue $\lambda_{n, i}$. Figure 1 shows the boundary curve for the (a) reactive and (b) nonreactive modes by setting the discriminant of the characteristic polynomial $P_{n}(\lambda)=0$. As can be seen, the dynamics is oscillatory in most of the parameter space except in a small wedge, the singularity of which is located at $\omega_{n} \tau=1 /(3 \sqrt{3})$ and $\gamma_{0}=8 /(3 \sqrt{3}) \omega_{n}$. At this point, the characteristic polynomial has a triple root at $\lambda=-\sqrt{3} \omega_{n}$.

The linearized system describing the reactive mode, $n=0$, is characterized by three parameters - the barrier frequency $\omega_{0}$, the damping constant $\gamma_{0}$, and the bath correlation time $\tau$ - that have the dimension of either a time or an inverse time. The three eigenvalues $\lambda_{0, i}$ also have the dimension of an inverse time. Then, it is convenient to express these quantities in terms of $\omega_{0}$, which sets the overall time scale, and the two dimensionless parameters

$$
\mu=\frac{\lambda_{0,0}}{\omega_{0}}
$$

and

$$
v^{2}=\frac{\lambda_{0,0}\left(1+\lambda_{0,0} \tau\right)}{\omega_{0}^{2} \tau}=\mu^{2}\left(1+\frac{1}{\mu \omega_{0} \tau}\right) .
$$

The parameter $\mu$ takes values between zero and one, while $v$ varies between $\mu$ and $\infty$. In the white noise limit, $\tau \rightarrow 0$ and consequently $v \rightarrow \infty$, and $\lambda_{n, 2} \rightarrow-\infty$, which means that excitations in this phase space direction decay infinitely fast. In this way, the phase space of the LE with white noise, which has dimension $2 d$ instead of $3 d$, is recovered. 

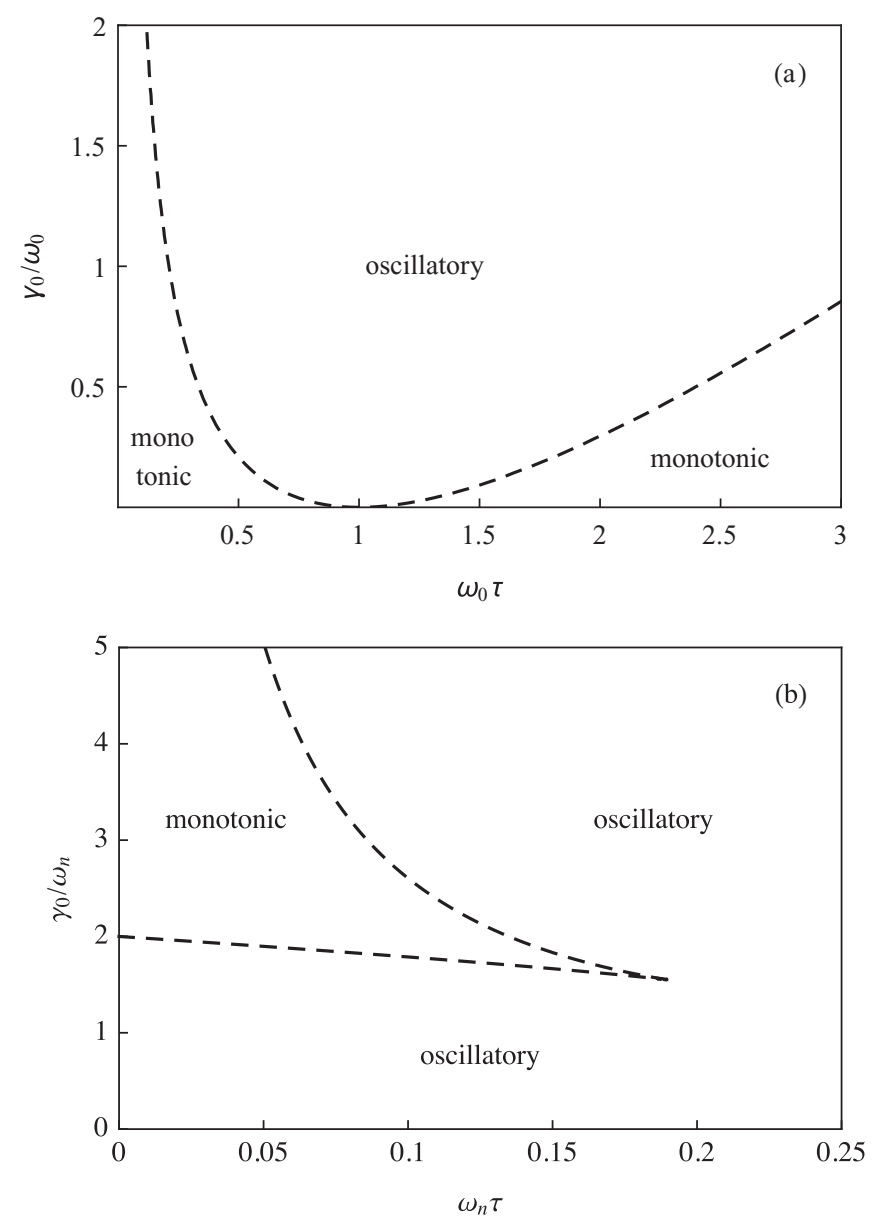

FIG. 1. Parameters leading to oscillatory and monotonic behavior for the stable directions of the linear generalized Langevin equation with exponential friction. The border between these two different behaviors, which is shown in dashed lines, is given by $\lambda_{n, 1}=\lambda_{n, 2}$ for (a) $n=0$ in the reactive mode and (b) $n=1,2, \ldots, d-1$ in the transversal modes.

In order to solve Eq. (16), we introduce the diagonal coordinates $z_{n, i}$, by decomposing the phase space vector

$$
\boldsymbol{u}_{n}=z_{n, 0} \tilde{\boldsymbol{u}}_{n, 0}+z_{n, 1} \tilde{\boldsymbol{u}}_{n, 1}+z_{n, 2} \tilde{\boldsymbol{u}}_{n, 2}
$$

in the basis of eigenvectors $\tilde{\boldsymbol{u}}_{n, i}$. In components, the transformation (24) reads

$$
\begin{aligned}
x_{n}= & z_{n, 0}+z_{n, 1}+z_{n, 2}, \\
v_{n}= & \lambda_{n, 0} z_{n, 0}+\lambda_{n, 1} z_{n, 1}+\lambda_{n, 2} z_{n, 2}, \\
\zeta_{n}= & {\left[\lambda_{n, 0}^{2}+\sigma_{n} \omega_{n}^{2}\right] z_{n, 0}+\left[\lambda_{n, 1}^{2}+\sigma_{n} \omega_{n}^{2}\right] z_{n, 1} } \\
& +\left[\lambda_{n, 2}^{2}+\sigma_{n} \omega_{n}^{2}\right] z_{n, 2} .
\end{aligned}
$$

Its inverse is given by

$$
\begin{aligned}
\left(\lambda_{n, i}\right. & \left.-\lambda_{n, j}\right)\left(\lambda_{n, i}-\lambda_{n, k}\right) z_{n, i} \\
& =\left[\lambda_{n, j} \lambda_{n, k}+\sigma_{n} \omega_{n}^{2}\right] x_{n}-\left(\lambda_{n, j}+\lambda_{n, k}\right) v_{n}+\zeta_{n},
\end{aligned}
$$

where the indices $i, j, k=0,1,2$ always take different values. In the new coordinates, the EoM (14) take the form

$$
\dot{z}_{n, i}=\lambda_{n, i} z_{n, i}+K_{n, i} f_{n}(\boldsymbol{x})+\frac{1}{F_{n, i}} \xi_{\alpha, n}(t),
$$

where the abbreviations $F_{n, i}=\left(\lambda_{n, i}-\lambda_{n, j}\right)\left(\lambda_{n, i}-\lambda_{n, k}\right)$ and $K_{n, i}=-\left(\lambda_{n, j}+\lambda_{n, k}\right) / F_{n, i}$ have been used.

\section{TIME-DEPENDENT INVARIANT MANIFOLDS}

The equations (27) can be solved by making a shift of origin to the relative coordinates $[40,45,46,51]$

$$
\Delta z_{n, i}(t)=z_{n, i}(t)-z_{n, i}^{\ddagger}(t), \quad i=0,1,2,
$$

where $z_{n, i}^{\ddagger}$ are the components of the TS trajectory, which is defined as

$$
z_{n, i}^{\ddagger}(t)=\frac{1}{F_{n, i}} S\left[\lambda_{n, i}, \xi_{\alpha, n} ; t\right],
$$

with the $S$ functionals

$$
S_{t^{\prime}}[\lambda, g ; t]=\left\{\begin{array}{l}
-\int_{t}^{\infty} g\left(t^{\prime}\right) e^{\lambda\left(t-t^{\prime}\right)} d t^{\prime}: \operatorname{Re} \lambda>0 \\
+\int_{-\infty}^{t} g\left(t^{\prime}\right) e^{\lambda\left(t-t^{\prime}\right)} d t^{\prime}: \operatorname{Re} \lambda<0
\end{array}\right.
$$

introduced in Refs. [40,51]. The subscript $t^{\prime}$ indicates the integration variable, and it will be omitted unless necessary to avoid ambiguities. The TS trajectory clearly depends on the realization $\alpha$ of the noise. It is the only trajectory that for a given noise sequence remains ("jiggling") in the vicinity of the saddle point for all times, whereas a typical trajectory will descend into either the reactant or product wells in the distant past or future.

From the fluctuation-dissipation relation given by Eq. (15) for the white noise source $\xi_{\alpha, n}$, the correlation functions for the components of the TS trajectory are found to be

$$
\begin{aligned}
\left\langle z_{0,0}^{\ddagger}(t) z_{0,0}^{\ddagger}(0)\right\rangle_{\alpha} & =\frac{k_{\mathrm{B}} T \gamma_{0}}{m \tau^{2} \lambda_{0,0} F_{0,0}^{2}} e^{-\lambda_{0,0} t}, \\
\left\langle z_{0,0}^{\ddagger}(t) z_{0, i}^{\ddagger}(0)\right\rangle_{\alpha} & =0, \\
\left\langle z_{0, i}^{\ddagger}(t) z_{0,0}^{\ddagger}(0)\right\rangle_{\alpha} & =\frac{2 k_{\mathrm{B}} T \gamma_{0}\left(e^{-\lambda_{0,0} t}-e^{\lambda_{0, i} t}\right)}{m \tau^{2}\left(\lambda_{0,0}+\lambda_{0, i}\right) F_{0,0} F_{0, i}}, \\
\left\langle z_{0, i}^{\ddagger}(t) z_{0, j}^{\ddagger}(0)\right\rangle_{\alpha} & =-\frac{2 k_{\mathrm{B}} T \gamma_{0} e^{\lambda_{0, i} t}}{m \tau^{2}\left(\lambda_{0, i}+\lambda_{0, j}\right) F_{0, i} F_{0, j}}, \\
\left\langle z_{n, k}^{\ddagger}(t) z_{n, l}^{\ddagger}(0)\right\rangle_{\alpha} & =-\frac{2 k_{\mathrm{B}} T \gamma_{0} e^{\lambda_{n, k} t}}{m \tau^{2}\left(\lambda_{n, k}+\lambda_{n, l}\right) F_{n, k} F_{n, l}}, \\
\left\langle z_{n^{\prime}, k}^{\ddagger}(t) z_{n^{\prime \prime}, l}^{\ddagger}(0)\right\rangle_{\alpha} & =0 \quad \text { if } n^{\prime} \neq n^{\prime \prime},
\end{aligned}
$$

where $n \geqslant 1 ; i, j=1,2 ; k, l=0,1,2$; and $t \geqslant 0$.

The EoM for the relative coordinates in Eq. (28) read

$$
\Delta \dot{z}_{n, i}=\lambda_{n, i} \Delta z_{n, i}+K_{n, i} f_{n}(\boldsymbol{x}) .
$$

They do not explicitly depend on the noise, though the influence of the stochastic driving does, however, appear implicitly through the time-dependent shift of origin to the TS trajectory [see Eq. (28)].

In the harmonic limit, $f_{n}(\boldsymbol{x})=0$, Eq. (32) are decoupled and can be easily solved as

$$
\Delta z_{n, i}(t)=\Delta z_{n, i}(0) e^{\lambda_{n, i} t}, \quad i=0,1,2 .
$$

The coordinates $\Delta z_{0,0}(t)$ and $\Delta z_{n, i}(t)$ [for $(n, i) \neq(0,0)$ ] have very different time dependence because $\lambda_{0,0}>0$ and $\operatorname{Re} \lambda_{n, i}<0: \Delta z_{0,0}(t)$ grows exponentially in time, whereas the remaining $\Delta z_{n, i}$ shrink. All trajectories that asymptotically 

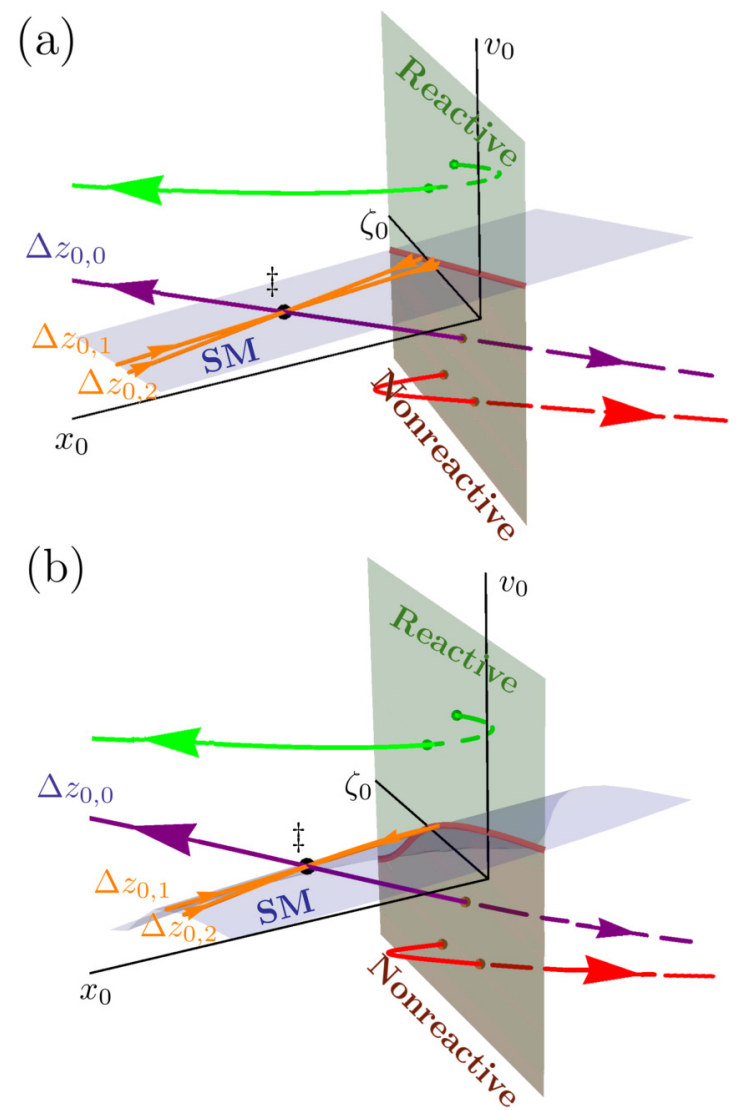

FIG. 2. Extended phase space of the generalized Langevin equation for a one-dimensional harmonic (a) and anharmonic (b) potential barrier. Black dot $(\$)$ : instantaneous position of the TS trajectory. Purple curve: unstable manifold. Light blue surface and orange trajectories within: stable manifold (SM). The dividing surface $\left(v_{0}-\zeta_{0}\right.$ plane) is partitioned into reactive (top green) and nonreactive (bottom brown) regions by the brown curve that indicates the intersection of the dividing surface with the stable manifold and defines the critical velocity $V^{\ddagger}\left(\zeta_{0}\right)$. Representative reactive (green) and nonreactive (red) trajectories intersect the dividing surface as indicated by green and brown dots, respectively.

approach the TS trajectory as $t \rightarrow \infty$ lie in the hyperplane $\Delta z_{0,0}=0$, which has dimension $3 d-1$. (It is thus a twodimensional plane for one-DOF systems.) This hyperplane is called the stable manifold of the TS trajectory. Similarly, trajectories that approach the TS trajectory backwards in time, as $t \rightarrow-\infty$, lie on the $\Delta z_{0,0}$ coordinate axis, i.e., the line $\Delta z_{n, i}=0$ for all $(n, i) \neq(0,0)$. This axis is the unstable manifold of the TS trajectory.

For the case of a system with one DOF, where the total dimension of phase space is 3 , the resulting geometry is sketched in Fig. 2(a). The instantaneous position of the TS trajectory is indicated by a black dot. Attached to it there is one unstable direction in which trajectories move away from the TS trajectory, and two stable directions from which neighboring trajectories approach the TS trajectory. The plane spanned by the two stable directions is the stable manifold. This plot captures the location of the manifolds at one instant of time. As the TS trajectory moves, the invariant manifolds will move with it. Because the eigenvectors $\tilde{\boldsymbol{u}}_{n, i}$ that determine the direction of the manifolds do not depend on time, this motion will be rigid, without rotation or distortion.

If the barrier potential is not harmonic, i.e., $f_{n}(\boldsymbol{x}) \neq 0$, the solution of the EoM (32) becomes more complicated. However, general theorems on dynamical systems [52,53] guarantee that the invariant manifolds persist in the presence of an anharmonic perturbation, as long as the perturbation is not too strong. They will, however, be distorted and will no longer be a straight line or a plane, as shown in Fig. 2(b). Moreover, the distortion is time dependent, so that the motion of the manifolds is no longer rigid. Nevertheless, the relative configuration of the manifolds will qualitatively remain as in the harmonic case, even though their precise location may be more difficult to determine.

The case of degenerate eigenvalues, where one of the matrices $\mathbf{M}_{\mathbf{n}}$ is not diagonalizable, is clearly exceptional. Nevertheless, it is worth discussing in detail because it introduces additional complications. If $\lambda_{n, 1}=\lambda_{n, 2}$ are equal, instead of two phase space directions in which the decay is given by $e^{\lambda_{n, 1} t}$ and $e^{\lambda_{n, 2} t}$, respectively, there will be two directions with decay $e^{\lambda_{n, 1} t}$ and $t e^{\lambda_{n, 1} t}$. Together they still span a two-dimensional invariant plane in phase space, so that the qualitative picture of the dynamics changes little. However, the quantitative description of the dynamics that was introduced above will fail, e.g., $F_{n, 1}=F_{n, 2}=0$ in Eq. (27). As a consequence, most of the intermediate results of the paper are invalid in the case of degenerate eigenvalues. The final rate corrections in Eqs. (53) and (61), however, are regular at the exceptional parameter values, as will be shown there. Therefore, these results must be correct for any values of the parameters.

The critical problem in rate theory is the correct identification of reactive trajectories. A careful examination of Fig. 2 shows that a knowledge of the invariant manifolds solves this problem: All trajectories outside the stable manifold will for large times leave the barrier region in the direction of the unstable manifold. Those that depart in the direction of positive $\Delta z_{0,0}$, which by Eq. (25) correspond to $x_{0}>0$ and therefore to the product region, are reactive, while those that leave in the direction of negative $\Delta z_{0,0}$ are nonreactive. The boundary between these two classes of trajectories is the stable manifold, which contains all those trajectories that approach the TS trajectory and never leave the barrier region. This scenario applies equally to higher-dimensional systems: The stable manifold is a hypersurface in phase space which separates the reactive and nonreactive regions. Crucially, both the location and the curvature are time dependent in a manner that is determined by the dynamics, without any arbitrary choices on the part of the investigator. The detailed dynamical information that is encoded in the stable manifold allows us to use a simple planar DS.

Note that, though the stable manifold separates trajectories with different future behavior, the unstable manifold cannot similarly be used to separate trajectories that came from the reactant or product regions in the past. The unstable manifold is only a curve in a phase space of at least three dimensions and has therefore a dimension too low to partition phase space into distinct regions. This complication, which occurs for white noise only in systems with several DOF's, is introduced by the presence of the auxiliary coordinates $\zeta_{n}$, that raise the dimension of phase space by one per DOF. 
Now we come to an important point. In order to carry out a rate calculation, we do not need to classify arbitrary trajectories as reactive or nonreactive. The flux expression (2) contains only trajectories that start at the DS $x_{0}^{\mathrm{DS}}=0$. As shown in Fig. 2, the stable manifold intersects the DS in the brown curve that can be regarded as the graph of a function $V^{\ddagger}\left(\zeta_{0}\right)$. This function will be called the critical velocity. In a multidimensional system, it will also depend on the bath mode coordinates $z_{\perp}=\left(\boldsymbol{x}_{\perp}, v_{\perp}, \zeta_{1}, \zeta_{2}, \ldots, \zeta_{d-1}\right)$. Though this is not explicit in our notation, the critical velocity depends on the realization $\alpha$ of the noise that determines the location of the stable manifold. Trajectories that start with velocities greater than $V^{\ddagger}\left(\zeta_{0}, z_{\perp}\right)$ are reactive, while trajectories with lower initial velocities are not. This is indicated by the red and green trajectories in Fig. 2, that start on the DS but on opposite sides of the stable manifold. The red trajectory begins with an initial velocity smaller than $V^{\ddagger}$ and it is therefore nonreactive, while the green trajectory is reactive because its initial velocity is sufficiently high. Note that both trajectories recross the DS: The red trajectory starts with positive velocity and therefore leaves the DS towards the product region, but then turns around and leaves towards the reactant side. Conversely, the green trajectory initially moves towards reactants, but ultimately moves off, towards the product side. This illustrates why traditional TST, using the criterion of $\chi^{\mathrm{TST}}\left(v_{0}\right)$ in Eq. (3), fails in the classification of these two trajectories. Finally, note that the stable manifold is only a local separatrix for reactivity. As a consequence, in the case of anharmonic barriers, the green (red) trajectory can dive to the product (reactant) well and then come back and cross the DS in the nonreactive (reactive) region shown in red (green). These nonlocal recrossings take place on much longer time scales; they correspond to subsequent crossings of the barrier. They are irrelevant for our purposes. Indeed, the assumption of moderate to strong friction implies that a trajectory that leaves the barrier region will thermalize in one of the wells before it can approach the barrier again. It is this assumption that allows us to neglect these nonlocal recrossings.

Because the critical velocity encodes all relevant information about reactivity, the characteristic function $\chi_{r}$ can be expressed in terms of it, as $\chi_{r}\left(v_{0}, \zeta_{0}, z_{\perp}\right)=\Theta\left[v_{0}-V^{\ddagger}\left(\zeta_{0}, z_{\perp}\right)\right]$. Despite its simplicity, this equation $\chi_{r}\left(v_{0}, \zeta_{0}, z_{\perp}\right)$ is still exact, and if we carry out the average over initial velocities in the flux in Eq. (2) we obtain the transmission factor

$$
\kappa=\left\langle e^{-m V^{\ddagger 2} / 2 k_{\mathrm{B}} T}\right\rangle_{\alpha \zeta \perp},
$$

where the average is extended over the realizations $\alpha$ of the noise, the initial conditions of the auxiliary coordinates $\zeta$, and the bath coordinates $z_{\perp}$. Equation (34) was originally derived to study systems driven by white noise in the harmonic limit in Ref. [43], but it has also been successfully used in Refs. $[20,45,46]$ to study anharmonic systems by adequately modifying the harmonic approximation to the critical velocity. In the following section, we describe how this task can be extended for colored noise using a perturbative scheme.

\section{THE CRITICAL VELOCITY}

To calculate the critical velocity $V^{\ddagger}\left(\zeta_{0}, z_{\perp}\right)$ in an anharmonic potential, the trajectory on the stable manifold that intersects the DS $x_{0}^{\mathrm{DS}}=0$ at the given values $\zeta_{0}$ and $z_{\perp}$ needs to be determined. If we take time $t=0$ as the moment of intersection, we are looking for a trajectory with IC $x_{0}(0)=$ $x_{0}^{\mathrm{DS}}=0$ and given values $\zeta_{0}(0)$ and $z_{\perp}(0)$ that lies on the stable manifold. The latter condition allows us to determine the unknown initial condition $v_{0}(0)$, which is the critical velocity $V^{\ddagger}\left(\zeta_{0}(0), z_{\perp}(0)\right)$.

As the initial conditions in the bath modes are known, only the reactive mode needs to be analyzed further. In terms of the diagonal coordinates $z_{0, n}$, the constraints on the IC read

$$
\begin{aligned}
& x_{0}(0)=0=z_{0,0}(0)+z_{0,1}(0)+z_{0,2}(0), \\
& \zeta_{0}(0)=\lambda_{0,0}^{2} z_{0,0}(0)+\lambda_{0,1}^{2} z_{0,1}(0)+\lambda_{0,2}^{2} z_{0,2}(0),
\end{aligned}
$$

where Eq. (35b) has been simplified with the help of Eq. (35a). These conditions can be used to eliminate two of the three initial values $z_{0, i}(0)$. At this point it is convenient to express the initial values of the stable DOF in terms of the unstable one as

$$
z_{n, i}(0)=\frac{\zeta_{n}(0)+\left(\lambda_{n, k}^{2}-\lambda_{n, 0}^{2}\right) z_{n, 0}(0)}{\lambda_{n, i}^{2}-\lambda_{n, k}^{2}},
$$

with $n \geqslant 0$, and $k=1,2$ for $i=2,1$. With these results, the critical velocity is obtained, after some algebra, as the intersection of the IC's with the stable manifold. Mathematically,

$$
\begin{aligned}
V^{\ddagger}=v_{0}(0) & =\lambda_{0,0} z_{0,0}(0)+\lambda_{0,1} z_{0,1}(0)+\lambda_{0,2} z_{0,2}(0) \\
& =\frac{1}{K_{0,0}} z_{0,0}(0)+\frac{1}{\lambda_{0,1}+\lambda_{0,2}} \zeta_{0}(0) .
\end{aligned}
$$

In general, this requires a detailed analysis of the dynamics. In the case of a harmonic barrier, however, the stable manifold is simply given by $\Delta z_{0,0}=0$, or $z_{0,0}=z_{0,0}^{\ddagger}$, and then the critical velocity is

$$
V^{\ddagger(0)}=\frac{1}{K_{0,0}} z_{0,0}^{\ddagger}(0)+\frac{1}{\lambda_{0,1}+\lambda_{0,2}} \zeta_{0}(0) .
$$

For the anharmonic case, we use perturbative expansions in terms of a small parameter $\varepsilon$ that measures the strength of the anharmonicity and is chosen as appropriate to a particular problem

$$
\begin{aligned}
V^{\ddagger} & =V^{\ddagger(0)}+\varepsilon V^{\ddagger(1)}+\varepsilon^{2} V^{\ddagger(2)}+\ldots, \\
z_{n, i}(0) & =z_{n, i}^{(0)}(0)+\varepsilon z_{n, i}^{(1)}(0)+\varepsilon^{2} z_{n, i}^{(2)}(0)+\ldots
\end{aligned}
$$

Substituting this into Eqs. (36) and (37) one obtains

$$
V^{\ddagger(k)}=\frac{1}{K_{0,0}} \Delta z_{0,0}^{(k)}(0)=\frac{1}{K_{0, i}} \Delta z_{0, i}^{(k)}(0),
$$

with $i=1,2$, because $\zeta_{0}(0)$ remains unchanged. Clearly, in the bath modes $\Delta z_{n, i}^{(k)}(0)=0$, for $n \geqslant 1$ and $k>0$ because the IC's in these modes are given. Thus, if we can determine the initial value $\Delta z_{0,0}^{(k)}(0)$ from the condition that the trajectory is on the stable manifold, we immediately obtain the corresponding correction to the critical velocity and to the initial values of the remaining coordinates, which in turn determine the trajectory completely.

To proceed, we use the formal solution of the EoM (32), which reads

$$
\Delta z_{0,0}(t)=C_{0,0} e^{\lambda_{0,0} t}+K_{0,0} S\left[\lambda_{0,0}, f_{0}(\boldsymbol{x}) ; t\right]
$$


for the unstable coordinate $\Delta z_{0,0}$. To incorporate the boundary condition that $z_{0,0}(t)$ remains bounded as $t \rightarrow \infty$, one must set $C_{0,0}=0$. The solutions for the stable coordinates $(n, i) \neq$ $(0,0)$ can be written as

$$
\Delta z_{n, i}(t)=\Delta z_{n, i}(0) e^{\lambda_{n, i} t}+K_{n, i} \bar{S}\left[\lambda_{n, i}, f_{n}(\boldsymbol{x}) ; t\right]
$$

in terms of the modified $S$ functional [46] $\bar{S}_{t^{\prime}}[\mu, g ; t]=$ $\int_{0}^{t} g\left(t^{\prime}\right) e^{\mu\left(t-t^{\prime}\right)} d t^{\prime}$, that satisfies the differential equation $\frac{d}{d t} \bar{S}[\mu, g ; t]=\mu \bar{S}[\mu, g ; t]+g(t)$ and the IC $\bar{S}[\mu, g ; 0]=0$.

The integral equations (41) and (42) represent only a formal solution to the EoM, since the unknown functions $\Delta z_{n, i}(t)$ occur on the right hand side. They are, however, well suited to a perturbative treatment: For a harmonic barrier, i.e., $f(x)=0$, we obtain the solutions $\Delta z_{0,0}(t)=0$ and $\Delta z_{n, i}(t)=$ $\Delta z_{n, i}(0) e^{\lambda_{n, i} t}$ for $(n, i) \neq(0,0)$. If we can insert this result into Eqs. (41) and (42), they will yield first order corrections to the stable manifold. The procedure can be iterated to obtain, in principle, corrections of arbitrarily high order.

In practice, solving the EoM (41) and (42) perturbatively requires an expansion not around $x_{0}=0$ but around the trajectory $\boldsymbol{X}(t)$ that is obtained in the harmonic limit, which equals

$$
\begin{aligned}
& X_{0}(t)=x_{0}^{\ddagger}(t)+\Delta z_{0,1}(0) e^{\lambda_{0,1} t}+\Delta z_{0,2}(0) e^{\lambda_{0,2} t}, \\
& X_{n}(t)=x_{n}^{\ddagger}(t)+\sum_{i=0}^{2} \Delta z_{n, i}(0) e^{\lambda_{n, i} t} \text { for } n \geqslant 1 .
\end{aligned}
$$

The coordinates $x_{n}$ can then be expanded as

$$
x_{n}(t)=X_{n}(t)+\varepsilon \Delta x_{n}^{(1)}(t)+\varepsilon^{2} \Delta x_{n}^{(2)}(t)+\ldots,
$$

where $\Delta x_{n}^{(k)}(t)=\Delta z_{n, 0}^{(k)}(t)+\Delta z_{n, 1}^{(k)}(t)+\Delta z_{n, 2}^{(k)}(t)$ are the corrections of order $k$ to the position $x_{n}$. Because the anharmonic force $f_{n}(\boldsymbol{x})$ is a known function of position, it can now be similarly expanded as

$$
f_{n}(\boldsymbol{x})=\varepsilon f_{n}^{(1)}+\varepsilon^{2} f_{n}^{(2)}+\ldots,
$$

where each term $f_{n}^{(k)}$ depends only on the harmonic trajectory $\boldsymbol{X}$ and corrections up to order $k-1$. It can therefore be used, together with Eqs. (41) and (42), to obtain the $k$ th order corrections to the trajectory as

$$
\begin{aligned}
& \Delta z_{0,0}^{(k)}(t)=K_{0,0} S\left[\lambda_{0,0}, f_{n}^{(k)} ; t\right], \\
& \Delta z_{n, i}^{(k)}(t)=\Delta z_{n, i}^{(k)}(0) e^{\lambda_{n, i} t}+K_{n, i} \bar{S}\left[\lambda_{n, i}, f_{n}^{(k)} ; t\right],
\end{aligned}
$$

where $(n, i) \neq(0,0)$. These yield in turn the correction $V^{\ddagger(k)}$ to the critical velocity and the force corrections $f_{n}^{(k+1)}$ of the next higher order, so that the process can continue.

\section{THE TRANSMISSION FACTOR}

The transmission factor in Eq. (5) can be expanded in terms of the perturbative parameter $\varepsilon$ by substituting Eq. (39a) in Eq. (34). This gives

$$
\kappa=\kappa^{(0)}+\varepsilon \kappa^{(1)}+\varepsilon^{2} \kappa^{(2)}+\ldots,
$$

where

$$
\begin{aligned}
\kappa^{(0)}= & \langle P\rangle_{\alpha \zeta \perp}, \\
\kappa^{(1)}= & -\frac{m}{k_{\mathrm{B}} T}\left\langle P V^{\ddagger(0)} V^{\ddagger(1)}\right\rangle_{\alpha \zeta \perp}, \\
\kappa^{(2)}= & \frac{m}{2 k_{\mathrm{B}} T}\left(\frac{m}{k_{\mathrm{B}} T}\left\langle P V^{\ddagger(0) 2} V^{\ddagger(1) 2}\right\rangle_{\alpha \zeta \perp}\right. \\
& \left.-2\left\langle P V^{\ddagger(0)} V^{\ddagger(2)}\right\rangle_{\alpha \zeta \perp}-\left\langle P V^{\ddagger(1) 2}\right\rangle_{\alpha \zeta \perp}\right),
\end{aligned}
$$

with the abbreviation $P=\exp \left(-\frac{m V^{\ddagger(0) 2}}{2 k_{\mathrm{B}} T}\right)$.

To evaluate Eqs. (48), we need to compute averages of the form $\langle P(\ldots)\rangle_{\alpha \zeta \perp}$, which we will call distorted correlation functions. To this end the factor $P$ appearing in Eqs. (48) can be absorbed into a modified covariance matrix. Full details of the procedure are given in Refs. $[45,46]$. Assume that the random variables $\left(w_{1}=V_{0}^{\ddagger}, w_{2}, w_{3}, \ldots, w_{m}\right)$ follow a multidimensional Gaussian distribution with zero mean and covariance matrix $\boldsymbol{\Sigma}$. Introduce a modified covariance matrix $\boldsymbol{\Sigma}_{0}$ that satisfies $\boldsymbol{\Sigma}_{0}^{-1}=\boldsymbol{\Sigma}^{-1}+\frac{m}{k_{\mathrm{B}} T} \mathbf{J}$, where $\mathbf{J}$ is a square matrix of size $d$ with $J_{1,1}=1$ and $J_{i, j}=0$ otherwise. By spelling out the averages as integrals over the Gaussian probability density function, we can show that $\langle P(\ldots)\rangle_{\alpha \zeta \perp}=\frac{\lambda_{0,0}}{\omega_{0}}\langle\ldots\rangle_{0}$, where $\langle\ldots\rangle_{0}$ denotes an average over a multidimensional Gaussian distribution with zero mean and the new matrix $\boldsymbol{\Sigma}_{0}$.

The matrix $\boldsymbol{\Sigma}_{0}$ is explicitly given by

$$
\boldsymbol{\Sigma}_{0}=\boldsymbol{\Sigma}-\frac{m}{k_{\mathrm{B}} T} \frac{\lambda_{0,0}^{2}}{\omega_{0}^{2}} \boldsymbol{\Sigma} \mathbf{J} \boldsymbol{\Sigma},
$$

which means in components that

$$
\left\langle w_{i} w_{j}\right\rangle_{0}=\left\langle w_{i} w_{j}\right\rangle_{\alpha \zeta \perp}-\frac{m}{k_{\mathrm{B}} T} \frac{\lambda_{0,0}^{2}}{\omega_{0}^{2}}\left\langle V^{\ddagger(0)} w_{i}\right\rangle_{\alpha \zeta \perp}\left\langle V^{\ddagger(0)} w_{j}\right\rangle_{\alpha \zeta \perp} .
$$

This allows us to compute the moments of the modified Gaussian distribution once those of the original Gaussian are known. Modified averages involving more than two factors can be obtained by Isserlis's theorem, e.g., $\left\langle w_{1} w_{2} w_{3} w_{4}\right\rangle_{0}=$ $\left\langle w_{1} w_{2}\right\rangle_{0}\left\langle w_{3} w_{4}\right\rangle_{0}+\left\langle w_{1} w_{3}\right\rangle_{0}\left\langle w_{2} w_{4}\right\rangle_{0}+\left\langle w_{1} w_{4}\right\rangle_{0}\left\langle w_{2} w_{3}\right\rangle_{0}$. This expression contains a sum over all possible pairings of the four factors. Other even order moments can be evaluated in a similar way, and all odd order moments are zero. In this way, the modified averages of arbitrary polynomials can be calculated.

For the leading order of the transmission factor for any multidimensional barrier, Eq. (48a) reduces to the well-known Grote-Hynes [54] result for a one-dimensional harmonic barrier:

$$
\kappa^{(0)}=\mu=\frac{\lambda_{0,0}}{\omega_{0}}\langle 1\rangle_{0}=\frac{\lambda_{0,0}}{\omega_{0}} .
$$

\section{RESULTS AND DISCUSSION}

\section{A. One-dimensional potential}

In this subsection, we present the results that we have obtained for a parabolic barrier with cubic and quartic anharmonicities, $U\left(x_{0}\right)=-m \omega_{0}^{2} x_{0}^{2} / 2+\varepsilon c_{3} x_{0}^{3} / 3+$ $\varepsilon^{2} c_{4} x_{0}^{4} / 4$. The force exerted by this potential is given by $-d U / d x_{0}=m \omega_{0}^{2} x_{0}+f\left(x_{0}\right)$, where $f\left(x_{0}\right) \equiv f_{0}\left(x_{0}\right)=$ $-\varepsilon c_{3} x_{0}^{2}-\varepsilon c_{4} x_{0}^{3}$. The first $f^{(k)}$ terms appearing in Eq. (46) 
are the coefficients in the expansion of the anharmonic force $f\left(X_{0}+\varepsilon \Delta x_{0}^{(1)}+\ldots\right)$, given by $f^{(1)}=-c_{3} X_{0}^{2}$ and $f^{(2)}=$ $-\left(2 c_{3} X_{0} \Delta x_{0}^{(1)}+c_{4} X_{0}^{3}\right)$. By substitution of these terms into Eqs. (40) and (46), one finally gets that the first and second order corrections to the critical velocity are given by

$$
\begin{gathered}
V^{\ddagger(1)}=-c_{3} S\left[\lambda_{0,1}, X_{0}^{2} ; 0\right], \\
V^{\ddagger(2)}=-2 c_{3} S\left[\lambda_{0,0}, X_{0} \Delta x_{0}^{(1)} ; 0\right]-c_{4} S\left[\lambda_{0,0}, X_{0}^{3} ; 0\right],
\end{gathered}
$$

with $\Delta x_{0}^{(1)}(t)=\Delta z_{0,0}^{(1)}(t)+\Delta z_{0,1}^{(1)}(t)+\Delta z_{0,2}^{(1)}(t)$. Then, the first order rate correction to Eq. (49) can be rewritten as

$$
\kappa^{(1)}=-\frac{m c_{3}}{k_{\mathrm{B}} T} \frac{\lambda_{0,0}}{\omega_{0}} S\left[\lambda_{0,0},\left\langle V^{\ddagger(0)} X_{0}^{2}\right\rangle_{0} ; 0\right]=0,
$$

which is zero because the correlation function is of third order in $V^{\ddagger(0)}$ and $X_{0}$. Similarly, all higher rate corrections of odd order vanish. As a consequence, the expansion (47) contains only even powers of the perturbation parameter $\varepsilon$. It will therefore yield an expansion in integer powers of $k_{\mathrm{B}} T$, rather than an expansion in powers $\sqrt{k_{\mathrm{B}} T}$, as one might expect at first sight.

The second order correction in Eq. (48c) to the rates has three terms, which can be computed by means of Isserlis's theorem. It will yield a sum of exponentially decaying terms, for which the $S$ functionals, which are short-hand notation for the integral (30), can be computed. The calculation is straightforward with the help of a computer algebra system, Mathematica [55] in our case, giving the rate correction

$$
\begin{aligned}
\kappa^{(2)}= & -\frac{c_{3}^{2} k_{\mathrm{B}} T}{6 m \omega_{0}^{6}} \frac{\mu\left(\mu^{2}-1\right)^{2}}{\left(\mu^{2}+v^{2}\right)\left[\mu^{4}+2 \mu^{2} v^{2}+4\left(v^{2}-\mu^{2}\right)\right]\left[\mu^{4}+\mu^{2} v^{2}+\left(v^{2}-\mu^{2}\right)\right]^{2}\left[4 \mu^{4}+2 \mu^{2} v^{2}+\left(v^{2}-\mu^{2}\right)\right]} \\
& \times\left[2\left(10 \mu^{4}+41 \mu^{2}+10\right) v^{10}+\left(110 \mu^{4}+329 \mu^{2}-12\right) \mu^{2} v^{8}+2\left(115 \mu^{4}+197 \mu^{2}-28\right) \mu^{4} v^{6}\right. \\
& \left.+2\left(115 \mu^{4}+22 \mu^{2}+8\right) \mu^{6} v^{4}+2\left(55 \mu^{4}-94 \mu^{2}+6\right) \mu^{8} v^{2}+5\left(4 \mu^{4}-17 \mu^{2}+4\right) \mu^{10}\right] \\
& -\frac{3 c_{4} k_{\mathrm{B}} T}{4 m \omega_{0}^{4}} \frac{\mu\left(\mu^{2}-1\right)^{2}\left(\mu^{2}+v^{2}\right)^{2}}{\left[\mu^{4}+\mu^{2} v^{2}+\left(v^{2}-\mu^{2}\right)\right]^{2}} .
\end{aligned}
$$

In the limit $v \rightarrow \infty$, which corresponds to white noise, this expression reduces to

$$
\kappa^{(2)}(\nu \rightarrow \infty)=-\frac{c_{3}^{2} k_{\mathrm{B}} T}{6 m \omega_{0}^{6}} \frac{\mu\left(1-\mu^{2}\right)^{2}}{\left(1+\mu^{2}\right)^{2}} \frac{\left(10+41 \mu^{2}+10 \mu^{4}\right)}{\left(2+5 \mu^{2}+2 \mu^{4}\right)}-\frac{3 c_{4} k_{\mathrm{B}} T}{4 m \omega_{0}^{4}} \frac{\mu\left(1-\mu^{2}\right)^{2}}{\left(1+\mu^{2}\right)^{2}} .
$$

Equations (53) and (54) were first derived, in somewhat more general if less explicit form, in Refs. [30,31]. Equation (54) was obtained by a method similar to this paper in Refs. [45,46]. As discussed in Sec. IV the rate corrections Eqs. (53) and (54) are nonsingular for all values of $\mu$ and $\nu$, even those where the matrix $\mathbf{M}_{\mathbf{0}}$ has a double eigenvalue and the intermediate steps of the derivation fail. Indeed, because $0<\mu<1$ and $v>\mu$, it is easy to see that none of the denominators can ever be zero.

The rate correction obtained from Eq. (53) is compared to the result of numerical simulations in Fig. 3. For the latter, classical trajectories are numerically propagated, using the algorithm described in Refs. [56,57], until their energy is far enough below the saddle point, so that they can be considered thermalized. To obtain converged results, the cutoff energy at which trajectories are considered to be thermalized on either reactant or product side must be chosen sufficiently low. In fact, it must be significantly lower than what would be required for a similar computation with white noise. This effect can be clearly seen in the top panel of the figure. An energy cutoff of $-3 k_{\mathrm{B}} T$ (blue plus symbols) is not enough to identify the reactive trajectories reliably, even in the harmonic limit, $c_{3}=0$, where the Grote-Hynes result (49) is exact (black horizontal line). This is due to the memory effect inherent in correlated noise: The friction force remembers that the trajectory came from the barrier top and therefore tends to push it back up. Thus, a lower energy cutoff has to be chosen. As can be seen in the same panel, the smaller the cutoff energy, the more accurate the results. Indeed, results are well converged for a cutoff energy of $-7 k_{\mathrm{B}} T$, and this value will be used in all further calculations. The converged transmission factors are in good agreement with the perturbative results. If $c_{3} \neq 0$, the potential has a minimum on one side of the barrier. The cutoff energy cannot be chosen below the minimum, or, conversely, for a given cutoff energy the coupling strength $c_{3}$ must be chosen such as to produce a sufficiently low minimum. For this reason, the data in the figure cover a smaller range of $c_{3}$ if the cutoff is lower.

Similar comments apply to Fig. 3(b), where the transmission factor is shown as a function of a quartic coupling strength $c_{4}$. Again, the numerical results decrease as the cutoff energy is lowered, finally converging to a limit that is in good agreement with the perturbative results if the coupling is not too strong.

Figure 4 shows the dependence of the transmission factor on the memory time. This dependence is strong, and more importantly it is largely accounted for by the harmonic approximation. Nevertheless, the deviation from the harmonic approximation also varies strongly with the memory time. The absolute value of the anharmonic correction is smallest in the white noise limit $\tau \rightarrow 0$. It grows for nonzero memory times, has a maximum at $\tau_{\min } \approx 4.5$, and then decreases again. This behavior is qualitatively well described by the leading order perturbation theory. The agreement between perturbation theory and simulation is excellent also in quantitative terms for memory times shorter than $\tau_{\min }$. For larger times, it is only approximate.

Similar results are shown in Fig. 5 for the transmission factor as a function of damping strength. The transmission factor 

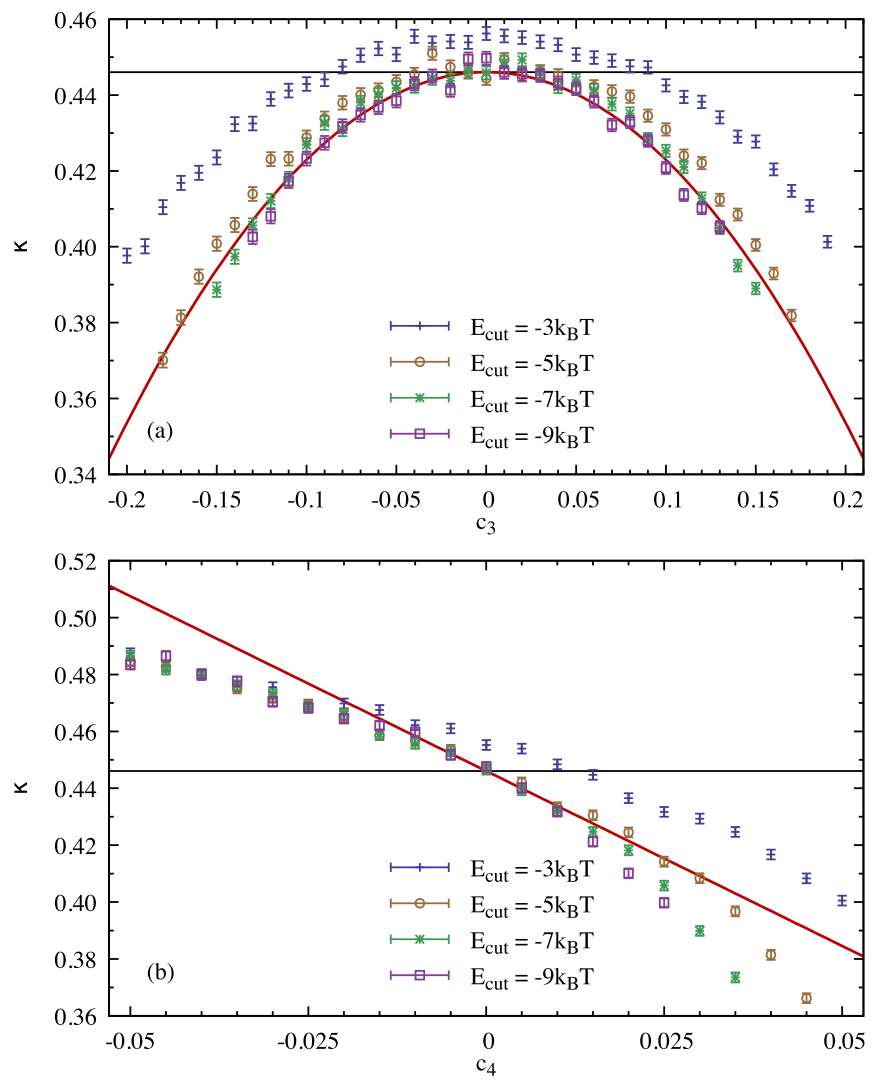

FIG. 3. Transmission factor for an anharmonic barrier with (a) cubic and (b) quartic perturbation. Horizontal black line: GroteHynes transmission factor given by Eq. (49). Red curve: leading order perturbation theory result obtained from the sum of Eqs. (49) and (53). Symbols: numerical simulation with $1 \sigma$ statistical error bars and different cutoff energies. Parameters used are equal to $m=1, \omega_{0}=1, \gamma_{0}=5, \tau=4, k_{\mathrm{B}} T=1$.

depends strongly on the strength of the damping, and again most of this dependence is accounted for by the harmonic approximation. The anharmonic correction is zero for $\gamma_{0}=0$, increases in magnitude for nonzero friction, then goes through a minimum and finally decreases again. Perturbation theory is in excellent agreement with the numerical results over the entire range of $\gamma_{0}$.

It should be kept in mind that the results are not physically meaningful in the limit of weak damping, because the rate theory outlined in Sec. II assumes that the rate is determined by spatial diffusion. As $\gamma_{0} \rightarrow 0$, a turnover to an energy diffusion limited rate will occur at a value of $\gamma_{0}$ that depends on the details of the potential well. It cannot therefore be stated in general how strong the damping has to be for the results of Fig. 5 to be applicable. For an assessment of the perturbative results, however, this question is not relevant.

Finally, let us remark that the one-dimensional version of the theory outlined here has been also successfully applied to more realistic chemical models [20]. Actually, we were able to accurately reproduce the reaction rates of the $\mathrm{LiNC} \rightleftharpoons$ LiCN isomerization reaction in the presence of an argon bath obtained using all-atom molecular dynamics by using a simple one-dimensional model defined along the minimum energy path of the molecule.

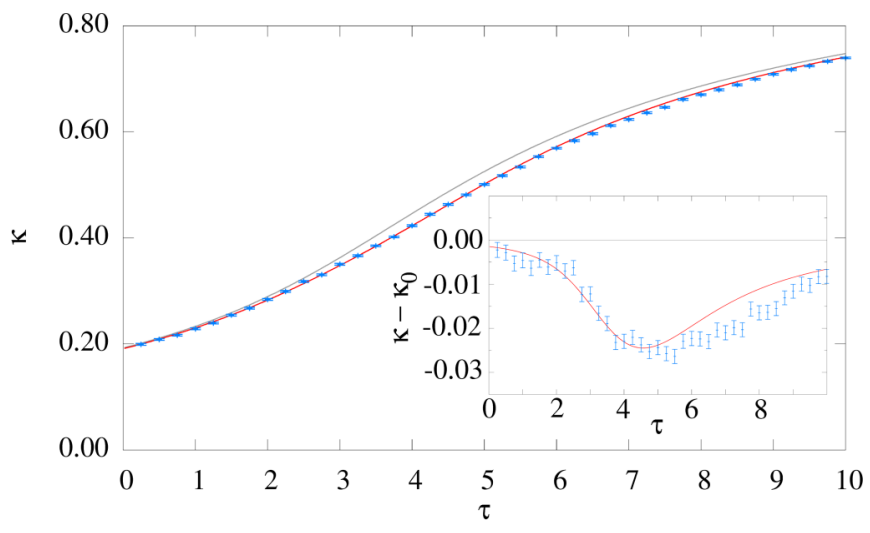

FIG. 4. Transmission factor as a function of memory time. Top black line: Grote-Hynes transmission factor given by Eq. (49). Bottom red line: leading order perturbation theory obtained from the sum of Eqs. (49) and (53). Blue symbols: numerical simulation with $1 \sigma$ error bars and cutoff energy $-7 k_{\mathrm{B}} T$. Inset: Deviation from the harmonic approximation. Parameters used are $m=1, \omega_{0}=1$, $\gamma_{0}=5, c_{3}=0.1, c_{4}=0, k_{\mathrm{B}} T=1$.

\section{B. The two-dimensional case}

Most chemical systems of interest have many DOF's, which can make their dynamics quite intricate. Accordingly, they cannot be described using simplified one-dimensional models. For the sake of simplicity, we will restrict our study to the two-DOF potential given by

$$
U\left(x_{0}, x_{1}\right)=-\frac{m \omega_{0}^{2}}{2} x_{0}^{2}+\frac{m \omega_{1}^{2}}{2} x_{1}^{2}+m c x_{0}^{2} x_{1}^{2} .
$$

This model potential does not have any minima, and therefore there is no well-defined barrier height. As we focus on the process of barrier crossing itself, rather than the ascent from the well to the barrier, this limitation is irrelevant for our current purposes. In spite of its simplicity, the model exhibits all the relevant geometric features and

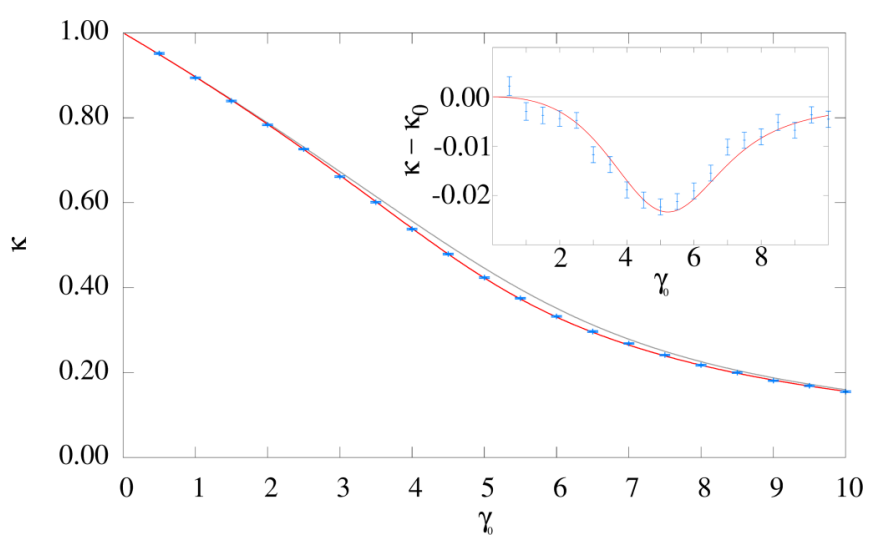

FIG. 5. Transmission factor as a function of damping. Top black line: Grote-Hynes transmission factor given by Eq. (49). Bottom red line: leading order perturbation theory obtained from the sum of Eqs. (49) and (53). Blue symbols: numerical simulation with $1 \sigma$ error bars and cutoff energy $-7 k_{\mathrm{B}} T$. Inset: Deviation from the harmonic approximation. Parameters used are $m=1, \omega_{0}=1, \tau=4, c_{3}=0.1$, $c_{4}=0, k_{\mathrm{B}} T=1$. 
allows an investigation both of the configuration of invariant manifolds and of their impact on the reaction rate in a multidimensional system. Thus, the techniques described here are essentially the same as those that are necessary to study anharmonic barriers with higher dimensionality, and the procedure presented here can be extended for systems of any dimension.

Reaction rates expressions for this potential have already been reported for the white noise case in Refs. [42,43,46]. Notice that the anharmonic coupling term is of even order in both coordinates and, consequently, corrections to the rate will appear at first order in the coupling constant $c$. The (extended) phase space associated with Eq. (55) has six dimensions, three more than for the case of one DOF (see previous subsection) and two more than the two-DOF case driven by white noise.

\section{The critical velocity}

To find the critical velocity of the trajectories lying at the DS, we have to solve Eqs. (41) and (42) with the IC's $x_{0}(0)=$ $x_{0}^{\mathrm{DS}}=0, \zeta_{0}(0), x_{1}(0), v_{1}(0)$, and $\zeta_{1}(0)$. The IC's $z_{0,1}(0)$ and $z_{0,2}(0)$ of the stable coordinates in the reactive DOF must be adapted in each step of perturbation theory in order to fulfill the conditions given by Eq. (36), from which it follows that $z_{1, i}(0)=\frac{\lambda_{1, j} \lambda_{1, k}-\omega_{1}^{2}}{F_{1, i}} x_{1}(0)+K_{1, i} v_{1}(0)+\frac{1}{F_{1, i}} \zeta_{1}(0) \quad$ with $i \neq j \neq k$.

If the coordinates

$$
x_{n}(t)=X_{n}(t)+c \Delta x_{n}^{(1)}(t)+c^{2} \Delta x_{n}^{(2)}(t)+\ldots
$$

are expanded in powers of the coupling constant $c$ and the force exerted by the potential in Eq. (55) is split into harmonic and anharmonic parts according to Eq. (11), we obtain the components of the anharmonic force:

$$
\begin{aligned}
f_{0} & =c f_{0}^{(1)}+c^{2} f_{0}^{(2)}+\ldots \\
& =-2 c X_{0} X_{1}^{2}-2 c^{2}\left(X_{1}^{2} \Delta x_{0}^{(1)}+2 X_{0} X_{1} \Delta x_{1}^{(1)}\right)+\ldots, \\
f_{1} & =c f_{1}^{(1)}+c^{2} f_{1}^{(2)}+\ldots \\
& =-2 c X_{0}^{2} X_{1}-2 c^{2}\left(X_{0}^{2} \Delta x_{1}^{(1)}+2 X_{0} X_{1} \Delta x_{0}^{(1)}\right)+\ldots
\end{aligned}
$$

The critical velocity in Eq. (39a) is similarly expanded as

$$
V^{\ddagger}=V^{\ddagger(0)}+c V^{\ddagger(1)}+c^{2} V^{\ddagger(2)}+\ldots
$$

We are now in a position to obtain explicit analytical expressions for the perturbative corrections to the critical velocity. Recall that the leading order term is given by the harmonic approximation to Eq. (58). Notice that it is given by Eq. (38). It is independent of the dimensionality of the system as in the absence of coupling between the different coordinates; i.e., when $c=0$ for the potential (55), the EoM (32) for the reactive DOF given by $n=0$ coincide with those for the one-DOF setting.

The first correction term for $\Delta z_{0,0}$ is given by $\Delta z_{0,0}^{(1)}(t)=$ $-2 K_{0,0} S\left[\lambda_{0,0}, X_{0} X_{1}^{2} ; t\right]$, which, combined with Eq. (58), gives the first order correction of the critical velocity:

$$
V^{\ddagger(1)}=\frac{1}{K_{0,0}} \Delta z_{0,0}^{(1)}(0)=-2 S\left[\lambda_{0,0}, X_{0} X_{1}^{2} ; 0\right] .
$$

To compute the second order perturbative term of the critical velocity, we also have to calculate the first order corrections to the stable coordinates, $\Delta z_{1,1}^{(1)}(t)$ and $\Delta z_{1,2}^{(1)}(t)$. Then, the second order correction to the critical velocity can be similarly obtained as

$$
\begin{aligned}
V^{\ddagger(2)}= & \frac{1}{K_{0,0}} \Delta z_{0,0}^{(2)}(0)=-2 S\left[\lambda_{0,0}, X_{1}^{2} \Delta x_{0}^{(1)}+2 X_{0} X_{1} \Delta x_{1}^{(1)} ; 0\right] \\
= & 4 S_{t}\left[\lambda_{0,0}, X_{1}^{2}(t)\left(K_{0,0} S\left[\lambda_{0,0}, X_{0} X_{1}^{2} ; t\right]+K_{0,1} S\left[\lambda_{0,0}, X_{0} X_{1}^{2} ; 0\right] e^{\lambda_{0,1} t}+K_{0,2} S\left[\lambda_{0,0}, X_{0} X_{1}^{2} ; 0\right] e^{\lambda_{0,2} t}+K_{0,1} \bar{S}\left[\lambda_{0,1}, X_{0} X_{1}^{2} ; t\right]\right.\right. \\
& \left.\left.+K_{0,2} \bar{S}\left[\lambda_{0,2}, X_{0} X_{1}^{2} ; t\right]\right)+2 X_{0}(t) X_{1}(t)\left(K_{1,0} \bar{S}\left[\lambda_{1,0}, X_{0}^{2} X_{1} ; t\right]+K_{1,1} \bar{S}\left[\lambda_{1,1}, X_{0}^{2} X_{1} ; t\right]+K_{1,2} \bar{S}\left[\lambda_{1,2}, X_{0}^{2} X_{1} ; t\right]\right) ; 0\right] . \quad(60)
\end{aligned}
$$

\section{The transmission factor}

Substituting the expansion of the critical velocity given by Eq. (58) in Eq. (47), we get an expansion of the transmission factor in the coupling constant, $c$, as $\kappa=\kappa^{(0)}+c \kappa^{(1)}+$ $c^{2} \kappa^{(2)}+\ldots$, where the coefficients $\kappa^{(i)}$ are given by Eqs. (48). Let us recall again here that the lowest order term of the transmission factor equals the Grote-Hynes expression (49). The higher order perturbative terms for the transmission factor are more complicated to calculate since they are formed by averages over the noise and the IC's that involve the harmonic approximation to the critical velocity, $V^{\ddagger(0)}$, and the random variables $X_{0}$ and $X_{1}$. Nevertheless, explicit expressions for them can be computed by applying Isserlis's theorem, as explained in Sec. IV.
It is important to recall that, for the computation of the transmission factor, $\left\langle V^{\ddagger(0)} X_{1}(t)\right\rangle_{0 \zeta \perp}=\left\langle X_{0}(t) X_{1}(s)\right\rangle_{0 \zeta \perp}=$ 0 since $X_{0}(t)$ and $X_{1}(t)$ depend on different components of the Gaussian noise $\xi_{\alpha, n}(t)$, which are not correlated. In particular, the presence of the factor $P$ in Eq. (48) has no influence on averages over the transverse DOF, while those in the reactive DOF are computed as in the one-DOF case (see above the corresponding subsection).

The first order correction to $\kappa^{(0)}$ can be calculated combining Eqs. (48b) and (59), yielding

$$
\kappa^{(1)}=-\frac{k T \mu\left(1-\mu^{2}\right)\left(\mu^{2}+v^{2}\right)}{\left[\mu^{4}+\mu^{2} v^{2}+\left(v^{2}-\mu^{2}\right)\right] m \Omega^{2} \omega_{0}^{4}},
$$



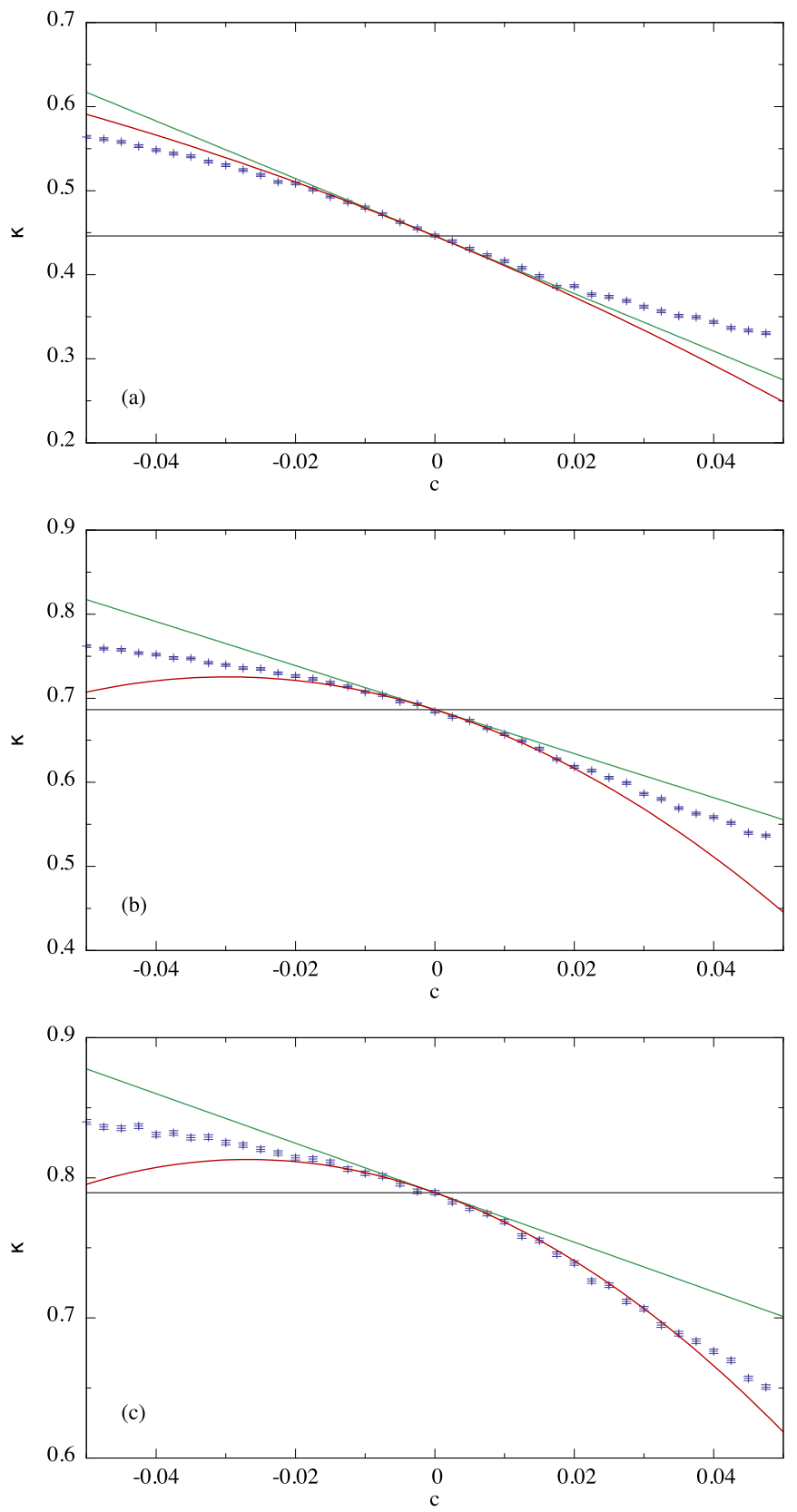

FIG. 6. Transmission factor for the two-dimensional model potential in Eq. (55) as a function of the coupling strength, $c$, for $m=1, \omega_{0}=1, \omega_{1}=0.5, \gamma_{0}=5, k_{\mathrm{B}} T=1, \tau=4$ (a), $\tau=8$ (b), and $\tau=12$ (c): numerical simulation results with $1 \sigma$ statistical error bars (blue symbols), harmonic (Grote-Hynes) approximation given by Eq. (49) (black horizontal line), perturbative results to first order obtained from the sum of Eqs. (49) and (61) (green straight line), and perturbative results to second order obtained from the sum of Eqs. (49), (61), and (48c) (red parabolic line).

where $\Omega=\omega_{1} / \omega_{0}$, which agrees with the white noise limit

$$
\kappa^{(1)}(\nu \rightarrow \infty)=-\frac{k T \gamma_{0} \mu^{2}}{\left(1+\mu^{2}\right) m \Omega^{2} \omega_{0}^{5}}
$$

obtained in Ref. [46], since $\gamma_{0}=\left(1-\mu^{2}\right) \omega_{1} / \mu$ in that limit. [The corresponding Eq. (85) in Ref. [46] contains a misprint.]
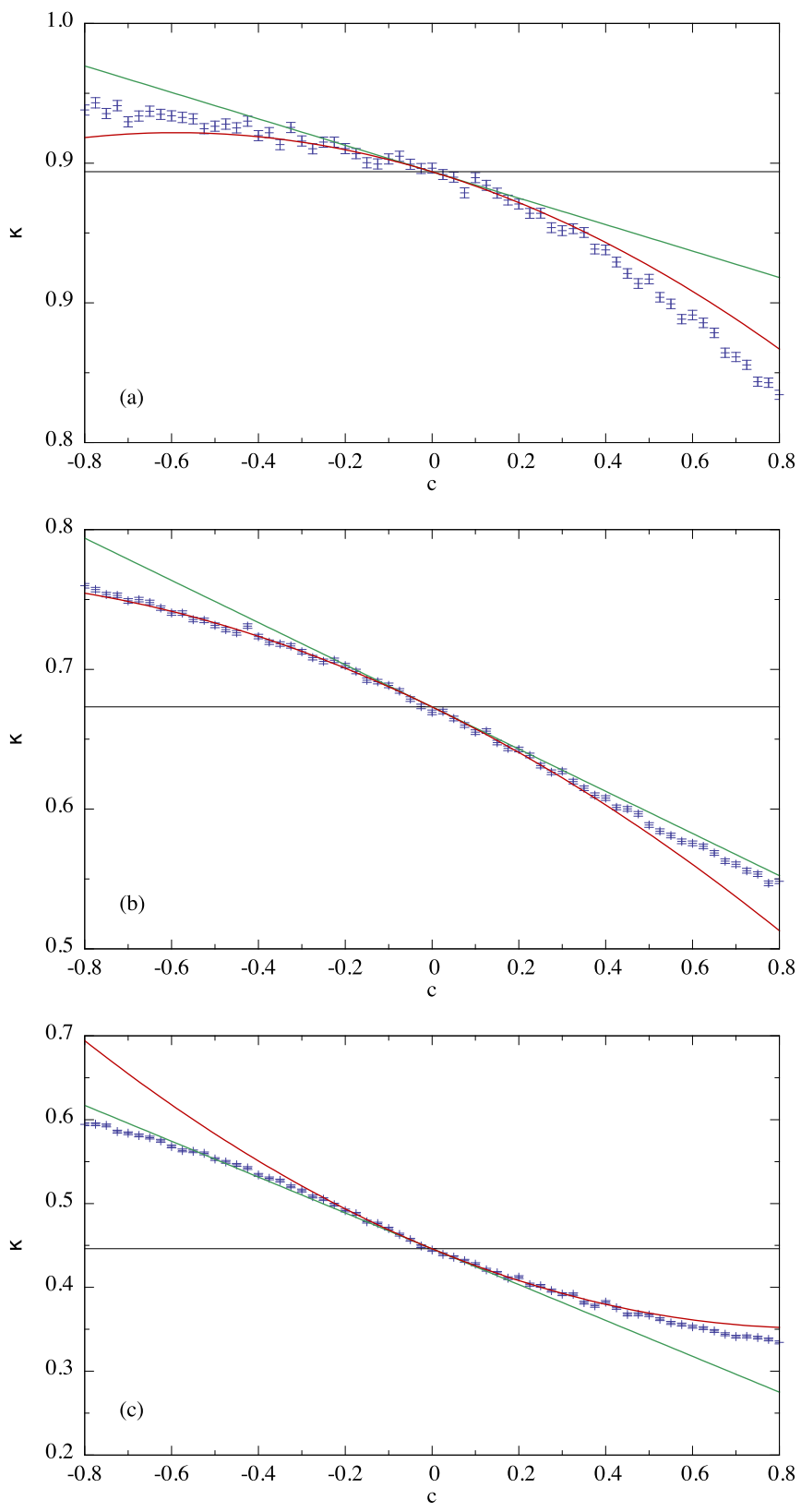

FIG. 7. Transmission factor for the two-dimensional model potential in Eq. (55) as a function of the coupling strength, $c$, for $m=1, \omega_{0}=1, \omega_{1}=2, \tau=4, k_{\mathrm{B}} T=1, \gamma_{0}=1$ (a), $\gamma_{0}=3$ (b), and $\gamma_{0}=5(\mathrm{c})$ : numerical simulation results with $1 \sigma$ statistical error bars (blue symbols), harmonic (Grote-Hynes) approximation given by Eq. (49) (black horizontal line), perturbative results to first order obtained from the sum of Eqs. (49) and (61) (green straight line), and perturbative results to second order obtained from the sum of Eqs. (49), (61), and (48c) (red parabolic line).

Once again, the rate correction given by Eq. (61) is nonsingular for all values of the parameters.

The second order correction of the transmission factor does not have such a simple expression, since $\kappa^{(2)}$ contains coupling terms that depend on sums and differences of $\lambda_{0, i}$ and $\lambda_{1, i}$ that cannot be simplified using the Vieta relations in Eq. (21). The explicit expression of $\kappa^{(2)}$ can be found in 
the Mathematica notebook included as Supplemental Material [58].

In Fig. 6 we show the transmission factor for the two-DOF model potential given by Eq. (55) as a function of the coupling constant, $c$, for three different values of the memory time $\tau$. Figure 7 shows similar results, but here the transverse frequency $\omega_{1}$ is larger than in Fig. 6, i.e., the reaction channel is narrower. In addition, in Fig. 7 the memory time is kept fixed and the damping strength $\gamma_{0}$ varies from one panel to the next. As for the one-dimensional case previously described, the most important contribution to the transmission factor is given by the Grote-Hynes expression (49). Similarly, it depends strongly both on the coupling strength and on the memory time, decreasing with the former and increasing with the latter. Our perturbative scheme gives excellent results for the two-DOF potential if the anharmonic coupling is not too strong. The agreement between theory and simulations is not as good for large values of the coupling constant. Nevertheless, our method stills provides good qualitative results.

As the transverse frequency $\omega_{1}$ increases, the dependence of the transmission factor on the coupling parameter gets weaker, as demonstrated by comparison of Figs. 6(a) and 7 (c), which differ only in the value of $\omega_{1}$. (Note that the range of the coupling strength is larger in Fig. 7 than in Fig. 6.) This can be well understood by looking at the first order correction to the transmission factor given by Eq. (61). That expression demonstrates that the first order correction to the transmission factor is proportional to $\Omega^{-2}$ and then to $\omega_{1}^{-2}$. Figure 7 demonstrates, furthermore, that our perturbative results agree with the numerical simulations even for quite strong anharmonicities if the transversal frequency becomes larger. Finally, Fig. 7 also shows that the transmission factor reduces with the damping strength, as it does in one DOF (see also Fig. 5).

\section{CONCLUDING REMARKS}

The computation of chemical reaction rates using TST and similar approaches is very common in the chemistry community. However, the results rendered by standard TST depend dramatically on the choice of an adequate DS. This is particularly important in reactions that take place in a solvent, where reactive trajectories can cross the DS more than once and, as a consequence, standard TST grossly overestimates the true reaction rate.
In this paper we present a method that overcomes the recrossing problem in anharmonic barriers with many dimensions. It identifies reactive trajectories precisely by computing the geometrical structures that divide the phase space into reactive and nonreactive parts. More specifically, all the information on the reactivity of the system is encoded in the stable manifold, the intersection of which with the DS defines a critical velocity that trajectories must exceed in order to be reactive. Notice that this procedure is independent of the selected DS as the stable manifold acts as a separatrix throughout the (extended) phase space. The intersection of the stable manifold with a different DS renders a different critical velocity but if a trajectory is reactive it will cross each DS with a velocity larger than the corresponding critical velocity. In any case, we have shown that the stable manifold defines a surface (in systems with one DOF) or a hypersurface (in systems with more than one DOF) that partitions the phase space as long as the potential energy surface has a saddle $\times$ center $\times$ center $\times \ldots \times$ center structure.

The method reported here is based on a perturbative scheme. It extends a previous series of studies [20,40-46] to the case of colored noise in higher dimensions. Likewise, its accuracy has been demonstrated by application to single exponential friction kernels with barriers with one or two degrees of freedom, though it can be extended to multiexponential kernels and higher dimensions, as the reaction is controlled by the same geometrical structures discussed here. Furthermore, it has also been successfully applied to calculate the reaction rates of a realistic molecular system [20]. Finally, it has enabled us to obtain analytic corrections to the Grote-Hynes expression for anharmonic multidimensional potentials, while providing at the same time a clear geometrical picture of the reaction mechanism.

\section{ACKNOWLEDGMENTS}

The research leading to these results has received funding from the Ministerio de Economía, Industria y Competitividad, Gobierno de España under Contract No. MTM2015-63914P, from ICMAT Severo Ochoa under Grant No. SEV-20150554, and from the European Union's Horizon 2020 research and innovation programme under Grant No. 734557. F.R. also acknowledges the financial support of the Programa Propio of the Universidad Politécnica de Madrid.
[1] R. Marcelin, Ann. Phys. 3, 120 (1915).

[2] M. G. Evans and M. Polanyi, Trans. Faraday Soc. 31, 875 (1935).

[3] E. Wigner, Trans. Faraday Soc. 34, 29 (1938).

[4] J. Horiuti, Bull. Chem. Soc. Japan 13, 210 (1938).

[5] M. Toller, G. Jacucci, G. DeLorenzi, and C. P. Flynn, Phys. Rev. B 32, 2082 (1985).

[6] B. Eckhardt, J. Phys. A 28, 3469 (1995).

[7] R. Hernandez and W. H. Miller, Chem. Phys. Lett. 214, 129 (1993).

[8] R. Hernandez, J. Chem. Phys. 101, 9534 (1994).
[9] C. Jaffé, D. Farrelly, and T. Uzer, Phys. Rev. A 60, 3833 (1999).

[10] C. Jaffé, D. Farrelly, and T. Uzer, Phys. Rev. Lett. 84, 610 (2000).

[11] W. S. Koon, M. W. Lo, J. E. Marsden, and S. D. Ross, Chaos 10, 427 (2000).

[12] C. Jaffé, S. D. Ross, M. W. Lo, J. Marsden, D. Farrelly, and T. Uzer, Phys. Rev. Lett. 89, 011101 (2002).

[13] T. Uzer, C. Jaffé, J. Palacián, P. Yanguas, and S. Wiggins, Nonlinearity 15, 957 (2002).

[14] T. Komatsuzaki and R. S. Berry, Adv. Chem. Phys. 123, 79 (2002). 
[15] E. Pollak, J. Chem. Phys. 85, 865 (1986).

[16] E. Pollak and P. Talkner, Phys. Rev. E 51, 1868 (1995).

[17] P. Pechukas and E. Pollak, J. Chem. Phys. 71, 2062 (1979).

[18] E. Pollak, M. S. Child, and P. Pechukas, J. Chem. Phys. 72, 1669 (1980).

[19] A. Allahem and T. Bartsch, J. Chem. Phys. 137, 214310 (2012).

[20] F. Revuelta, T. Bartsch, P. L. Garcia-Muller, R. Hernandez, R. M. Benito, and F. Borondo, Phys. Rev. E 93, 062304 (2016).

[21] P. Hänggi, P. Talkner, and M. Borkovec, Rev. Mod. Phys. 62, 251 (1990).

[22] P. Pechukas, in Dynamics of Molecular Collisions, Part B, edited by W. H. Miller (Plenum, New York, 1976), pp. 269-322.

[23] D. Chandler, J. Chem. Phys. 68, 2959 (1978).

[24] J. P. Bothma, J. B. Gilmore, and R. H. McKenzie, New J. Phys. 12, 055002 (2010).

[25] D. Polli, P. Altoè, O. Weingart, K. M. Spillane, C. Manzoni, D. Brida, G. Tomasello, G. Orlandi, P. Kukura, R. A. Mathies, M. Garavelli, and G. Cerullo, Nature (London) 467, 440 (2010).

[26] W. T. Coffey, Y. P. Kalmykov, and J. T. Waldrom, The Langevin Equation, World Scientific Series in Contemporary Chemical Physics Vol. 14 (World Scientific, Singapore, 2005).

[27] C. C. Martens, J. Chem. Phys. 116, 2516 (2002).

[28] J. B. Conway, A Course in Functional Analysis, 2nd ed., Graduate Texts in Mathematics Vol. 96 (Springer, New York, 1990).

[29] A. N. Drozdov and P. Talkner, Phys. Rev. E 54, 6160 (1996).

[30] E. Pollak and P. Talkner, Phys. Rev. E 47, 922 (1993).

[31] P. Talkner and E. Pollak, Phys. Rev. E 47, R21 (1993).

[32] P. Talkner, Chem. Phys. 180, 199 (1994).

[33] E. Pollak and J. Ankerhold, J. Chem. Phys. 138, 164116 (2013).

[34] E. Pollak and R. Ianconescu, J. Chem. Phys. 140, 154108 (2014).

[35] R. Ianconescu and E. Pollak, J. Chem. Phys. 143, 104104 (2015).

[36] E. Pollak and R. Ianconescu, J. Phys. Chem. A 120, 3155 (2016).
[37] R. Zwanzig, J. Stat. Phys. 9, 215 (1973).

[38] A. M. Frishman and E. Pollak, J. Chem. Phys. 98, 9532 (1993).

[39] P. L. García-Müller, F. Borondo, R. Hernandez, and R. M. Benito, Phys. Rev. Lett. 101, 178302 (2008).

[40] T. Bartsch, R. Hernandez, and T. Uzer, Phys. Rev. Lett. 95, 058301 (2005).

[41] T. Bartsch, T. Uzer, and R. Hernandez, J. Chem. Phys. 123, 204102 (2005).

[42] T. Bartsch, T. Uzer, J. M. Moix, and R. Hernandez, J. Chem. Phys. 124, 244310 (2006).

[43] T. Bartsch, T. Uzer, J. M. Moix, and R. Hernandez, J. Phys. Chem. B 112, 206 (2008).

[44] R. Hernandez, T. Uzer, and T. Bartsch, Chem. Phys. 370, 270 (2010).

[45] F. Revuelta, T. Bartsch, R. M. Benito, and F. Borondo, J. Chem. Phys. 136, 091102 (2012).

[46] T. Bartsch, F. Revuelta, R. M. Benito, and F. Borondo, J. Chem. Phys. 136, 224510 (2012) [a misprint in Eq. (85) of this reference is corrected in Eq. (58) of the present paper] .

[47] M. Ferrario and P. Grigolini, J. Math. Phys. 20, 2567 (1979).

[48] P. Grigolini, J. Stat. Phys. 27, 283 (1982).

[49] F. Marchesoni and P. Grigolini, J. Chem. Phys. 78, 6287 (1983).

[50] T. Bartsch, J. Chem. Phys. 131, 124121 (2009).

[51] S. Kawai, A. D. Bandrauk, C. Jaffé, T. Bartsch, J. Palacián, and T. Uzer, J. Chem. Phys. 126, 164306 (2007).

[52] J. Duan, An Introduction to Stochastic Dynamics (Cambridge University, Cambridge, England, 2015).

[53] L. Arnold, Random Dynamical Systems (Springer, Berlin, 1998).

[54] R. F. Grote and J. T. Hynes, J. Chem. Phys. 73, 2715 (1980).

[55] S. Wolfram, The Mathematica Book (Wolfram Media, Champaign, 2003).

[56] E. Hershkovitz, J. Chem. Phys. 108, 9253 (1998).

[57] E. Hershkovitz and R. Hernandez, J. Phys. Chem. A 105, 2687 (2001).

[58] See Supplemental Material at http://link.aps.org/supplemental/ 10.1103/PhysRevE.99.052211 for explicit expressions of the transmission factor in the Mathematica notebook. 